Giuseppe Cavaliere, Luca De Angelis, Anders Rahbek and A.M.Robert Taylor

A Comparison of Sequential and Information-based Methods for Determining the Co-integration Rank in Heteroskedastic VAR Models

Quaderni di Dipartimento

Serie Ricerche 2013, n. 4 ISSN 1973-9346

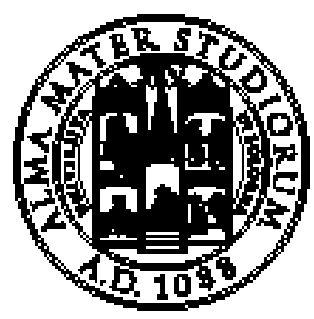

ALMA MATER STUDIORUM UNIVERSITA DI BOLOGNA

Dipartimento di Scienze Statistiche "Paolo Fortunati" 


\title{
A Comparison of Sequential and Information-based Methods for Determining the Co-integration Rank in Heteroskedastic VAR Models*
}

\author{
Giuseppe Cavaliere ${ }^{a}$, Luca De Angelis ${ }^{a}$, Anders Rahbek ${ }^{b}$ and A.M.Robert Taylor ${ }^{c}$ \\ ${ }^{a}$ Department of Statistical Sciences, University of Bologna \\ ${ }^{b}$ Department of Economics, University of Copenhagen
}

${ }^{c}$ School of Economics and Granger Centre for Time Series Econometrics, University of Nottingham

March 2013

\begin{abstract}
In this paper we investigate the behaviour of a number of methods for estimating the cointegration rank in VAR systems characterized by heteroskedastic innovation processes. In particular we compare the efficacy of the most widely used information criteria, such as AIC and BIC, with the commonly used sequential approach of Johansen (1996) based around the use of either asymptotic or wild bootstrap-based likelihood ratio type tests. Complementing recent work done for the latter in Cavaliere, Rahbek and Taylor (2013, Econometric Reviews, forthcoming), we establish the asymptotic properties of the procedures based on information criteria in the presence of heteroskedasticity (conditional or unconditional) of a quite general and unknown form. The relative finite-sample properties of the different methods are investigated by means of a Monte Carlo simulation study. For the simulation DGPs considered in the analysis, we find that the BICbased procedure and the bootstrap sequential test procedure deliver the best overall performance in terms of their frequency of selecting the correct co-integration rank across different values values of the co-integration rank, sample size, stationary dynamics and models of heteroskedasticity. Of these the wild bootstrap procedure is perhaps the more reliable overall since it avoids a significant tendency seen in the BIC-based method to over-estimate the co-integration rank in relatively small sample sizes.
\end{abstract}

Keywords: Co-integration; Wild bootstrap; Trace statistic; Information criteria; Rank determination; Heteroskedasticity.

J.E.L. Classifications: C30, C32.

\footnotetext{
${ }^{*}$ Cavaliere, Rahbek and Taylor thank the Danish Council for Independent Research, Sapere Aude - DFF Advanced Grant (Grant nr: 12-124980) for financial support. Cavaliere and De Angelis thank the Italian Ministry of Education, University and Research (MIUR) for financial support through the PRIN project "Multivariate statistical models for risk assessment". Correspondence to: Robert Taylor, School of Economics, University of Nottingham, Nottingham, NG7 2RD, U.K. E-mail: robert.taylor@nottingham.ac.uk
} 


\section{Introduction}

It is now well-known that sequential procedures based on the asymptotic (pseudo-) likelihood ratio [PLR] test of Johansen $(1988,1991,1996)$ for determining the co-integration rank of a VAR system of variables integrated of order one [denoted I(1)] can have quite poor finite sample properties; see, in particular, Johansen (2002), and the references therein. As a consequence, it is not surprising that a number of recent papers have investigated the use of alternative methods for determining the co-integration rank.

Information-based methods are widely used for econometric model selection and therefore provide an appealing alternative to approaches based on Neyman-Pearson type tests for determining the co-integration rank. A significant body of research on co-integration analysis has focused on the issue of co-integration rank estimation based on the use of standard information criteria. Under conditions which, among other things, rule out conditional or unconditional heteroskedasticity in the shocks, Aznar and Salvador (2002) and Kapetanios (2000) demonstrate the weak consistency (formally defined in Section 3) of approaches based on the familiar BIC (Rissanen, 1978; Schwarz, 1998) and HQC (Hannan and Quinn, 1979), outlining the conditions which need to hold on the associated penalty functions for this to obtain. Using Monte Carlo simulation, Wang and Bessler (2005) present results which suggest that the performance of a BIC-based approach is close to that of the approach based on PLR (trace) tests and tends to outperform the corresponding approach based on the AIC (Akaike, 1974). Indeed, Kapetanios (2004) establishes the inconsistency of the AIC-based approach, deriving its asymptotic distribution and showing that the resulting estimate of the co-integration rank displays a severe upward bias. Interestingly, Baltagi and Wang (2007) conduct a co-integration analysis on 165 data sets used in published studies. They find that the percentage of agreement between procedures based on $\mathrm{AIC}, \mathrm{HQC}$, and $\mathrm{BIC}$ is quite low at below $60 \%$, suggesting an apparent divergence in the co-integration rank suggested by the different information criteria.

Many key macroeconomic and financial variables appear to be characterized by permanent changes in unconditional volatility (see, e.g., McConnell and Perez Quiros, 2000; van Dijk, Osborn and Sensier, 2002; Sensier and van Dijk, 2004), and/or the presence of conditionally heteroskedastic shocks (see, e.g., Gonçalves and Kilian, 2004). Several authors have shown that traditional co-integration tests can display significant upward size distortions in the presence of conditionally heteroskedasticity (Lee and Tse, 1996; Cavaliere, Rahbek and Taylor, 2010a [CRT2010a, hereafter]) or non-stationary heteroskedasticity (Cavaliere, Rahbek and Taylor, 2010b [CRT2010b, hereafter]). Specifically, CRT2010b show that the sequential PLR method of Johansen (1996) is no longer valid, even asymptotically, in the presence of permanent changes in the error variance. In response to this, Cavaliere, Rahbek and Taylor (2012, 2013) [CRT2012 and CRT2013, respectively, hereafter] show that this can be rectified by using a proper bootstrap implementation of Johansen's sequential method. Specifically, they show that when (i) the bootstrap samples are constructed using the restricted parameter estimates of the underlying VAR model which obtain under the reduced rank null hypothesis, and (ii) the bootstrap shocks are generated according to the so-called wild bootstrap re-sampling scheme, then the bootstrap 
sequential procedure is consistent, in the sense that the probability of selecting a rank smaller than the true rank converges to zero and the probability of overestimating the true rank is bounded by the chosen significance level.

The asymptotic validity of the aforementioned information criteria based approaches to selecting the co-integration rank have only been established under the assumption of i.i.d. shocks. Cheng and Phillips (2012) show that the semi-parametric variants of standard information criteria approaches to selecting the co-integration rank (including the AIC, BIC and HQC procedures) proposed by Cheng and Phillips (2009) remain weakly consistent for the the co-integrating rank (under the same rate conditions on the penalty term) even if the shocks display time-varying heteroskedasticity of the form considered in CRT2010b. However, so far as we are aware, the properties of the fully parametric information criteria based procedures mentioned above have not been analysed for the cases of either conditionally or unconditionally heteroskedastic shocks.

The aim of this paper is to fill this gap in the literature by analysing and comparing the methods outlined above for determining the co-integration rank in an I(1) VAR system driven by heteroskedastic shocks. We make two distinct contributions to the literature. First, and for both the case of stationary conditional heteroskedasticity and non-stationary unconditional heteroskedasticity, we provide sufficient conditions for the information criteria to be weakly consistent as the sample size diverges. Second, we use Monte Carlo simulation methods to compare the performance of the procedures in cases where the innovations display time-varying behaviour in either their conditional or unconditional variances. Our simulation study provides some important insights into the finite-sample behaviour of the different methods of rank determination under the forms of conditional and unconditional heteroskedasticity considered.

The remainder of the paper is organized as follows. Section 2 describes the co-integrated VAR model with heteroskedastic innovations. Section 3 outlines the co-integration rank determination methods considered in our analysis. In Section 4 we analyse the large sample properties of the various methods considered. The results from our Monte Carlo simulation study are reported in Section 5 . Section 6 concludes the paper. Proofs are relegated to the Appendix.

In the following: $\stackrel{p}{\rightarrow}$ is used to denote convergence in probability, as $T \rightarrow \infty ; x:=y$ to indicate that $x$ is defined by $y ; I_{k}$ denotes the $k \times k$ identity matrix, $0_{k}$ the $k$-vector of zeroes and $0_{j \times k}$ the $j \times k$ matrix of zeroes; for any square matrix, $A,|A|$ is used to denote its determinant and $\|A\|$ the norm $\|A\|^{2}:=\operatorname{tr}\left\{A^{\prime} A\right\}$; for any vector, $x,\|x\|$ denotes the usual Euclidean norm, $\|x\|:=\left(x^{\prime} x\right)^{1 / 2}$.

\section{The Heteroskedastic Co-integrated VAR Model}

We consider the $p$-dimensional process $\left\{X_{t}\right\}$ which satisfies the $k$ th order reduced rank vector autoregressive (VAR) model:

$$
\Delta X_{t}=\alpha \beta^{\prime} X_{t-1}+\sum_{i=1}^{k-1} \Gamma_{i} \Delta X_{t-i}+\alpha \rho^{\prime} D_{t}+\phi d_{t}+\varepsilon_{t}, \quad t=1, \ldots, T
$$


where $X_{t}:=\left(X_{1 t}, \ldots, X_{p t}\right)^{\prime}$ and where the initial values, $X_{1-k}, \ldots, X_{0}$, are taken to be fixed in the statistical analysis. In the context of (2.1), CRT2012 define the 'I $(1, r)$ conditions' to hold where the characteristic polynomial associated with (2.1) has $p-r$ roots equal to 1 with all other roots lying outside the unit circle, and where $\alpha$ and $\beta$ have full column rank $r$.

The deterministic variables in 2.1 are taken to satisfy one of the following cases (see, e.g., Johansen, 1996): (i) $D_{t}=0, d_{t}=0$ (no deterministic component); (ii) $D_{t}=1, d_{t}=0$ (restricted constant); or (iii) $D_{t}=t, d_{t}=1$ (restricted linear trend). The innovation process $\varepsilon_{t}:=\left(\varepsilon_{1 t}, \ldots, \varepsilon_{p t}\right)^{\prime}$ is assumed to satisfy one of the following three assumptions outlined in CRT2013:

Assumption $\mathcal{V}$ The innovations $\left\{\varepsilon_{t}\right\}$ are independent and identically distributed with zero mean and a variance matrix $\Sigma$ of full-rank and satisfy the condition $\mathrm{E}\left\|\varepsilon_{t}\right\|^{4} \leq K<\infty$.

Assumption $\mathcal{V}$ ' With respect to the filtration $\mathcal{F}_{t}$, where $\mathcal{F}_{t-1} \subseteq \mathcal{F}_{t}$ for $t=\ldots,-1,0,1,2, \ldots$, the innovations $\left\{\varepsilon_{t}\right\}$ form a martingale difference sequence and satisfy $\mathrm{E}\left\|\varepsilon_{t}\right\|^{4} \leq K<\infty$ and the global homoskedasticity condition:

$$
\frac{1}{T} \sum_{t=1}^{T} \mathrm{E}\left(\varepsilon_{t} \varepsilon_{t}^{\prime} \mid \mathcal{F}_{t-1}\right) \stackrel{p}{\rightarrow} \Sigma>0 .
$$

Assumption $\mathcal{V}$ " The innovations $\left\{\varepsilon_{t}\right\}$ are defined as $\varepsilon_{t}=\sigma_{t} z_{t}$, where $z_{t} \sim$ i.i.d. $\left(0, I_{p}\right)$ with $\mathrm{E}\left\|z_{t}\right\|^{4} \leq$ $K<\infty$ and the matrix $\sigma_{t}$ is non-stochastid ${ }^{1}$ and satisfies $\sigma_{t}:=\sigma(t / T)$ for all $t=1, \ldots, T$, where $\sigma(\cdot) \in \mathcal{D}_{\mathbb{R}^{p \times p}}[0,1]$. Furthermore, $\Sigma(u):=\sigma(u) \sigma(u)^{\prime}$ is assumed to be positive definite for all $u \in[0,1]$.

Remark 1 As pointed out by CRT2013, Assumption $\mathcal{V}$ represents the basic assumption on $\varepsilon_{t}$ considered by Johansen (1996) with the Gaussianity assumption relaxed to a fourth-order moment requirement. Assumption $\mathcal{V}$ ' is introduced by CRT2010a for dealing with innovation processes that are serially uncorrelated and potentially conditionally heteroskedastic. In particular, Assumption $\mathcal{V}$ ' allows for, among other things: multivariate stable GARCH-type models and the autoregressive stochastic volatility models considered in Gonçalves and Kilian (2004), and models with deterministic or periodic heteroskedasticity. The condition in $(2.2)$ of Assumption $\mathcal{V}$ ' implies the so-called global stationarity (see, e.g., Davidson, 1994, pp. 454-455) which allows the conditional (and, consequently, unconditional) variance of $\varepsilon_{t}$ to be time-varying, provided that it is asymptotically stable over all possible fixed fraction of the data (see Remark 2.1 in CRT2010a). Assumption $\mathcal{V}$ " allows the elements of the innovation covariance matrix to display a countable number of jumps, provided that $\Sigma_{t}:=\sigma_{t} \sigma_{t}^{\prime}$ is bounded. Therefore, for example, denoting the $(i, j)$ th element of $\Sigma(u)$ by $\Sigma_{i j}(u)$, the case of a single break at time $\lfloor\tau T\rfloor$ in the covariance $\mathrm{E}\left(\varepsilon_{i t} \varepsilon_{j t}\right)$ obtains for $\Sigma_{i j}(u)=\Sigma_{i j}^{0}+\left(\Sigma_{i j}^{1}-\Sigma_{i j}^{0}\right) \mathbb{I}(u \geq \tau)$.

Remark 2 In the case where the $\mathrm{I}(1, r)$ conditions are satisfied and either Assumption $\mathcal{V}$ or $\mathcal{V}^{\prime}$ holds, $X_{t}$ is I(1) with co-integration rank $r$ such that the co-integration relations $\beta^{\prime} X_{t}-E\left(\beta^{\prime} X_{t}\right)$ are stationary $($ Assumption $\mathcal{V}$ ) or globally stationary (Assumption $\mathcal{V}$ '). In general, the co-integration

\footnotetext{
${ }^{1}$ As noted by CRT2013, the requirement that $\sigma_{t}$ is non-stochastic is only made to simplify the analysis and can be generalised to allow for cases where $\sigma(\cdot)$ is stochastic and independent of $z_{t}$; see Remark 2.2 of CRT2010b for further details.
} 
relations are not stationary under Assumption $\mathcal{V}$ ", since $\sigma_{t}$ is time-varying. However, $\beta^{\prime} X_{t}-E\left(\beta^{\prime} X_{t}\right)$ is free of stochastic trends and, thus, can be considered as stable (CRT2010b).

\section{Co-integration Rank Determination Methods}

In this section we briefly outline the methods for the co-integration rank determination considered in this paper. In addition to the well-known sequential likelihood ratio test of Johansen (1996) and its bootstrap counterpart (CRT2012 and CRT2013), we consider the most widely used information criteria (BIC, AIC, and HQC).

\subsection{Sequential (standard and bootstrap) Likelihood Ratio Tests}

Assuming knowledge of the lag length parameter $k$ in $(2.1$, the well-known PLR test of Johansen (1996) for the hypothesis of co-integration rank (less than or equal to) $r$ in (2.1), denoted $H(r)$, against $H(p)$, rejects for large values of the trace statistic,

$$
Q_{r, T}:=-T \sum_{i=r+1}^{p} \log \left(1-\hat{\lambda}_{i}\right),
$$

where $\hat{\lambda}_{1}>\ldots>\hat{\lambda}_{p}$ are the largest $p$ solutions to the eigenvalue problem,

$$
\left|\lambda S_{11}-S_{10} S_{00}^{-1} S_{01}\right|=0
$$

where $S_{i j}:=T^{-1} \sum_{t=1}^{T} R_{i t} R_{j t}^{\prime}, i, j=0,1$, with $R_{0 t}$ and $R_{1 t}$ respectively denoting $\Delta X_{t}$ and $\left(X_{t-1}^{\prime}, D_{t}\right)^{\prime}$, corrected (by OLS) for $\Delta X_{t-1}, \ldots, \Delta X_{t-k+1}$ and $d_{t}$. The sequential testing procedure based on $Q_{r, T}$ consists of, starting with $r=0$, testing iteratively $H(r)$ against $H(p)$, for $r=0, \ldots, p-1$, until, for a given value of $r$, the asymptotic $p$-value associated with $Q_{r, T}$, exceeds a chosen (marginal) significance level. In what follows we will denote this estimator as $\hat{r}_{P L R}$.

The analogous bootstrap estimator of the co-integrating rank, denoted $\hat{r}_{B L R}$ in what follows, obtained from the bootstrap analogue of the foregoing sequential procedure is proposed in CRT2012 and CRT2013 and iterates over the following steps, starting from $r=0$ :

(i) Estimate model (2.1) under $H(r)$ using Gaussian pseudo maximum likelihood yielding the estimates $\hat{\beta}^{(r)}, \hat{\alpha}^{(r)}, \hat{\rho}^{(r)}, \widehat{\Gamma}_{1}^{(r)}, \ldots ., \widehat{\Gamma}_{k-1}^{(r)}$ and $\phi^{(r)}$, together with the corresponding residuals, $\hat{\varepsilon}_{r, t} \cdot{ }^{2}$

(ii) Check that the equation $\left|\hat{A}^{(r)}(z)\right|=0$, with $\hat{A}^{(r)}(z):=(1-z) I_{p}-\hat{\alpha}^{(r)} \hat{\beta}^{(r) \prime} z-\sum_{i=1}^{k-1} \hat{\Gamma}_{i}^{(r)}(1-z) z^{i}$, has $p-r$ roots equal to 1 and all other roots outside the unit circle. If so, proceed to step (iii).

\footnotetext{
${ }^{2}$ The estimates $\hat{\beta}^{(r)}$ need to be defined as $\hat{\beta}^{(r)}:=\hat{V} K_{p}^{(r)}$, where $K_{p}^{(r)}:=\left(I_{r}, 0_{r \times(p-r)}\right)^{\prime}$ is a selection matrix indexed by $r$ and $p$, and, when deterministic terms are included, $\hat{\beta}^{\#(r)}:=\left(\hat{\beta}^{(r) \prime}, \hat{\rho}^{(r) \prime}\right)^{\prime}=\hat{V} K_{p+1}^{(r)}$. Here $\hat{V}=\left[\hat{v}_{1}, \ldots, \hat{v}_{p}\right]$, normalized by $\hat{V}^{\prime} S_{11} \hat{V}=I_{p}$, denotes the eigenvectors corresponding to the ordered eigenvalues $\hat{\lambda}_{1}>\ldots>\hat{\lambda}_{p}$ which solve the determinantal equation in (3.1). See CRT2012 for further details.
} 
(iii) Construct the bootstrap sample recursively from

$$
\Delta X_{r, t}^{*}=\hat{\alpha}^{(r)} \hat{\beta}^{(r) \prime} X_{r, t-1}^{*}+\sum_{i=1}^{k-1} \widehat{\Gamma}_{i}^{(r)} \Delta X_{r, t-i}^{*}+\hat{\alpha}^{(r)} \hat{\rho}^{(r) \prime} D_{t}+\hat{\phi}^{(r)} d_{t}+\varepsilon_{r, t}^{*}, t=1, \ldots, T
$$

initialised at $X_{r, j}^{*}=X_{j}, j=1-k, \ldots, 0$, and with the $T$ bootstrap errors $\varepsilon_{r, t}^{*}$ generated using the re-centred residuals, $\hat{\varepsilon}_{r, t}^{c}:=\hat{\varepsilon}_{r, t}-T^{-1} \sum_{i=1}^{T} \hat{\varepsilon}_{r, i}$, for either:

(a) the i.i.d. bootstrap, such that $\varepsilon_{r, t}^{*}:=\hat{\varepsilon}_{r, \mathcal{U}_{t}}^{c}$, where $\mathcal{U}_{t}, t=1, \ldots, T$ is an i.i.d. sequence of discrete uniform distributions on $\{1,2, \ldots, T\}$, or

(b) the wild bootstrap, where for each $t=1, \ldots, T, \varepsilon_{r, t}^{*}:=\hat{\varepsilon}_{r, t}^{c} w_{t}$, where $w_{t}, t=1, \ldots, T$, is an i.i.d. $\mathrm{N}(0,1)$ sequence.

(iv) Using the bootstrap sample, $\left\{X_{r, t}^{*}\right\}$, and denoting by $\hat{\lambda}_{1}^{*}>\ldots>\hat{\lambda}_{p}^{*}$ the ordered solutions to the bootstrap analogue of the eigenvalue problem in (3.1), compute the bootstrap LR statistic $Q_{r, T}^{*}:=-T \sum_{i=r+1}^{p} \log \left(1-\hat{\lambda}_{i}^{*}\right)$. Define the corresponding $p$-value as $p_{r, T}^{*}:=1-G_{r, T}^{*}\left(Q_{r, T}\right)$, $G_{r, T}^{*}(\cdot)$ denoting the conditional (on the original data) cdf of $Q_{r, T}^{*}$.

(v) If $p_{r, T}^{*}$ exceeds the significance level, $\eta$, set $\hat{r}_{B L R}=r$, otherwise repeat steps (i)-(iv) testing the null of rank $(r+1)$ against rank $p$ if $r+1<p$, or set $\hat{r}_{B L R}=p$ if $r+1=p$.

CRT2013 show that, in the presence of heteroskedasticity in the innovation process, the procedure based on the wild bootstrap significantly outperforms its i.i.d. bootstrap analogue. Consequently, we will only report simulation results in what follows for the wild bootstrap procedure; i.e., option (b) of step (iii) of the algorithm.

\subsection{Information Criteria}

The general form of the information criterion we consider in this paper is given by $\operatorname{IC}(r)=-2 \ell_{T}(r)+$ $p_{T}(r)$, where $\ell_{T}(r)$ is the maximised log-likelihood function under rank $r$ and $p_{T}(r)=c_{T} \pi(r)$ denotes the penalty function which depends on the number of parameters $\pi(r)$ and on the term $c_{T}$ which may depend on the sample size $T$.

Information criteria for determining the co-integration rank can thus be computed using the residual covariance matrix $\hat{\Sigma}^{(r)}$ estimated from the conventional reduced rank regression outlined in the previous subsection (see, e.g., Johansen, 1996) ${ }^{3}$ In particular, up to a constant term which does not depend on $r$, the maximised log-likelihood is given by $\ell_{T}(r)=-\frac{T}{2} \log \left|\hat{\Sigma}^{(r)}\right|$ where

$$
\left|\hat{\Sigma}^{(r)}\right|=\left|S_{00}\right| \prod_{i=1}^{r}\left(1-\hat{\lambda}_{i}\right)
$$

\footnotetext{
${ }^{3}$ Again knowledge of the lag length parameter $k$ in 2.1 is therefore also assumed in the context of the information criterion-based methods which follow.
} 
and $\hat{\lambda}_{1}>\ldots>\hat{\lambda}_{r}$ are the $r$ largest ordered eigenvalues obtained solving the determinantal equation in (3.1). Consequently, the general criterion used to assess co-integration rank is given by

$$
\mathrm{IC}(r):=T \log \left|\hat{\Sigma}^{(r)}\right|+c_{T} \pi(r)
$$

where $\pi(r)=r(2 p-r)$ when no deterministic component is involved, $\pi(r)=r(2 p-r+1)$ in the case of restricted constant, and $\pi(r)=r(2 p-r+1)+p$ in the restricted trend case. Different values of the coefficient $c_{T}$ yield different information criteria through the resulting penalty function, $p_{T}=c_{T} \pi(r)$. The most widely used are BIC, HQC and AIC where $c_{T}=\log T, 2 \log \log T$, and 2, respectively, thereby yielding:

$$
\begin{gathered}
\operatorname{BIC}(r):=T \log \left|\hat{\Sigma}^{(r)}\right|+(\log T) \pi(r), \\
\operatorname{HQC}(r):=T \log \left|\hat{\Sigma}^{(r)}\right|+2(\log \log T) \pi(r),
\end{gathered}
$$

and

$$
\operatorname{AIC}(r):=T \log \left|\hat{\Sigma}^{(r)}\right|+2 \pi(r) .
$$

The co-integration rank estimator is then given, in generic form, by

$$
\hat{r}_{\mathrm{IC}}:=\arg \min _{r=0,1, \ldots, p} \mathrm{IC}(r) .
$$

With an obvious notation, the resulting co-integration rank estimators corresponding to the BIC, HQC and AIC will be denoted $\hat{r}_{\mathrm{BIC}}, \hat{r}_{\mathrm{HQC}}$ and $\hat{r}_{\mathrm{AIC}}$.

\section{Asymptotic Analysis}

In this section we discuss and compare the asymptotic properties of the rank determination methods outlined in the previous section. In doing so, we complement the asymptotic analysis in CRT2012 and CRT2013 on (standard and bootstrap) sequential likelihood ratio testing and provide new results on the large-sample properties of information criteria in the presence of conditional and unconditional heteroskedasticity.

In what follows we let $r_{0}$ denote the true co-integration rank and, commensurately, in what follows we will often refer to the case where the parameters of (2.1) satisfy the $\mathrm{I}\left(1, r_{0}\right)$ conditions.

\subsection{Sequential (standard and bootstrap) Likelihood Ratio Tests}

It is known from results provided in Johansen (1996) and CRT2010a that the estimator of the cointegration rank obtained using the sequential procedure based on Johansen's asymptotic PLR test is consistent under i.i.d. shocks and conditionally heteroskedastic shocks satisfying Assumption $\mathcal{V}^{\prime}$, but that this is not the case when the shocks are unconditionally heteroskedastic, as in Assumption $\mathcal{V}$ ". For convenience we summarise these results in the following theorem. 
Theorem 1 Let $\left\{X_{t}\right\}$ be generated as in (2.1) with the parameters satisfying the $I\left(1, r_{0}\right)$ conditions. Then, under either Assumption $\mathcal{V}$ or $\mathcal{V}$ ', for a chosen significance level $\eta, \lim _{T \rightarrow \infty} P\left(\hat{r}_{P L R}=r\right)=0$ for all $r=0,1, \ldots, r_{0}-1 ; \lim _{T \rightarrow \infty} P\left(\hat{r}_{P L R}=r_{0}\right)=1-\eta$ for $r=r_{0}$ and $\lim _{T \rightarrow \infty} P\left(\hat{r}_{P L R}=r\right) \leq \eta$ for all $r=r_{0}+1, \ldots, p$. Under Assumption $\mathcal{V} "$, for any chosen significance level, $\lim _{T \rightarrow \infty} P\left(\hat{r}_{P L R}=r\right)=0$ for all $r=0,1, \ldots, r_{0}-1$.

Taken together, the results in Theorem 1 imply that the sequential procedure based on the asymptotic PLR tests (using standard asymptotic critical values) will never underestimate the co-integration rank in large samples. However, as shown in CRT2010b, this procedure will likely over-estimate the true co-integration rank, even in large samples, when unconditional heteroskedasticity of the form given in Assumption $\mathcal{V}$ "is present in the shocks, owing to the dependence of the limiting null distribution of the PLR statistic, $Q_{r_{0}, T}$, on nuisance parameters relating to the pattern of heteroskedasticity present. CRT2013 show that a consistent procedure can, however, be obtained by using the bootstrap tests from Section 3.1. CRT2013 demonstrate that, under Assumptions $\mathcal{V}$ and $\mathcal{V}$ ', the sequential procedure based on either the i.i.d. or wild bootstrap algorithm is consistent, but that only the latter bootstrap algorithm delivers a consistent sequential procedure under Assumption $\mathcal{V}$ ". These results relating to the bootstrap procedures are summarized in the following theorem.

Theorem 2 Let $\left\{X_{t}\right\}$ be generated as in (2.1) with the parameters satisfying the $I\left(1, r_{0}\right)$ conditions. Moreover, suppose that the limiting nonzero eigenvalues from (3.1) are distinct. Then,

(i) under either Assumption $\mathcal{V}$ or $\mathcal{V}$ ' and for either the i.i.d. or wild bootstrap re-sampling design in step (iii), for a chosen significance level $\eta, \lim _{T \rightarrow \infty} P\left(\hat{r}_{B L R}=r\right)=0$ for all $r=0,1, \ldots, r_{0}-1$; $\lim _{T \rightarrow \infty} P\left(\hat{r}_{B L R}=r_{0}\right)=1-\eta$ for $r=r_{0} ;$ and $\lim _{T \rightarrow \infty} P\left(\hat{r}_{B L R}=r\right) \leq \eta$ for all $r=r_{0}+1, \ldots, p ;$

(ii) under Assumption $\mathcal{V}$ ", for the wild bootstrap re-sampling design in step (iii)(b), for a chosen significance level $\eta, \lim _{T \rightarrow \infty} P\left(\hat{r}_{B L R}=r\right)=0$ for all $r=0,1, \ldots, r_{0}-1 ; \lim _{T \rightarrow \infty} P\left(\hat{r}_{B L R}=r_{0}\right)=$ $1-\eta$ for $r=r_{0}$; and $\lim _{T \rightarrow \infty} P\left(\hat{r}_{B L R}=r\right) \leq \eta$ for all $r=r_{0}+1, \ldots, p$.

\subsection{Information Criteria}

It is well-known (see, inter alia, Paulsen, 1984; Nielsen, 2006) that the BIC and HQC, but not the AIC, provide weakly consistent $\mathrm{H}^{4}$ estimates of the lag order $k$ in both pure $\mathrm{I}(0)$ and pure $\mathrm{I}(1)$ finiteorder VAR models driven by either i.i.d. or martingale difference shocks, provided $c_{T}$ in the penalty function analogous to that given in $(3.2)$ is such that $c_{T} \rightarrow \infty$ and $c_{T} / T \rightarrow 0$ as $T \rightarrow \infty$. Aznar and Salvador (2002) and Kapetanios (2000) show that the same conditions on $c_{T}$ ensure that $\hat{r}_{\text {BIC }}$ and $\hat{r}_{\mathrm{HQC}}$, as defined in Section 3.2, are weakly consistent for the co-integration rank in 2.1) in the case of where the shocks are i.i.d. (Assumption $\mathcal{V}$ ), but that this does not hold for $\hat{r}_{\text {AIC }}$ which is inconsistent.

\footnotetext{
${ }^{4} \mathrm{An}$ estimator $T_{n}$ is defined to be weakly consistent if it converges in probability to the true value of the unknown parameter $\theta$; i.e. $T_{n} \stackrel{p}{\rightarrow} \theta$.
} 
In Theorem 3 we now extend the results in Aznar and Salvador (2002) and Kapetanios (2000) to provide sufficient conditions on the penalty term that guarantee consistency under conditional and unconditional heteroskedasticity of the form given in Assumptions $\mathcal{V}$ ' and $\mathcal{V}$ '.

Theorem 3 Let $\left\{X_{t}\right\}$ be generated as in (2.1) with the parameters satisfying the $I\left(1, r_{0}\right)$ conditions. Then, under either Assumption $\mathcal{V}, \mathcal{V}$ ', or $\mathcal{V} "$, it holds that, as $T \rightarrow \infty$ :

(i) for $r>r_{0}, \operatorname{Prob}\left(I C(r)>I C\left(r_{0}\right)\right) \rightarrow 1$, provided $c_{T} \rightarrow \infty$;

(ii) for $r<r_{0}, \operatorname{Prob}\left(I C(r)>I C\left(r_{0}\right)\right) \rightarrow 1$, provided $c_{T} / T \rightarrow 0$.

Hence, if $\frac{c_{T}}{T}+\frac{1}{c_{T}} \rightarrow 0, \hat{r}_{I C} \stackrel{p}{\rightarrow} r_{0}$.

Remark 3 An immediate corollary of the results in Theorem 3 is that the BIC and HQC based estimators, $\hat{r}_{\mathrm{BIC}}$ and $\hat{r}_{\mathrm{HQC}}$, respectively, are weakly consistent for the co-integration rank while the corresponding AIC estimator, $\hat{r}_{\text {AIC }}$, is not because its penalty function does not satisfy the rate conditions on $c_{T}$ stated in the theorem. These results hold regardless of whether the innovations satisfy Assumption $\mathcal{V}, \mathcal{V}$ ', or $\mathcal{V}$ ". Notice that because AIC violates the rate condition required in (i) it will be an asymptotically upward biased estimator of the co-integration rank regardless of whether Assumption $\mathcal{V}, \mathcal{V}$ ', or $\mathcal{V} "$ holds.

Remark 4 It is seen from the results in Theorem 3 that the conditions required on $c_{T}$ to ensure the weak consistency of the BIC and HQC based co-integration rank estimators in the presence of conditional and unconditional heteroskedasticity are precisely the same as shown to be required for consistency under i.i.d. shocks by Aznar and Salvador (2002) and Kapetanios (2000). This is because the consistency of these estimators derives from the asymptotic order of the $\hat{\lambda}_{i}, i=1, \ldots, p$, rather than from their exact limits. Since the former is not influenced by the presence of heteroskedasticity in the innovations, the $\hat{r}_{\mathrm{BIC}}$ and $\hat{r}_{\mathrm{HQC}}$ estimators remain consistent under Assumptions $\mathcal{V}$ ' and $\mathcal{V}$ '. However, we anticipate that the finite sample behaviour of these procedures are likely to be affected by both the limiting distribution of $T \hat{\lambda}_{i}$, for $i=r_{0}+1, \ldots, p$, and the heteroskedastic form of the innovation process. This will be investigated further in the next section.

\section{$5 \quad$ Numerical Results}

Using Monte Carlo simulation we investigate the finite sample performances of the methods for estimating the co-integration rank outlined in Section 3 under both homoskedastic models and models with conditional or unconditional heteroskedasticity.

As our simulations DGP we will consider an extended version of the simulation DGP used in CRT2013, given by the following $\operatorname{VAR}(2)$ process of dimension $p=4$ :

$$
\Delta X_{t}=\alpha \beta^{\prime} X_{t-1}+\Gamma_{1} \Delta X_{t-1}+\varepsilon_{t}, \quad t=1, \ldots, T
$$


with $\varepsilon_{t}$ a martingale difference sequence (see below for specification details), $X_{0}=\Delta X_{0}=0$, and for sample sizes $T \in\{50,100,200,400\}$. The long-run parameter vectors are set to

$$
\beta^{\prime}:=\left[\begin{array}{llll}
1 & 0 & 0 & 0 \\
0 & 1 & 0 & 0
\end{array}\right] \text { and } \alpha^{\prime}:=\left[\begin{array}{cccc}
a & 0 & 0 & 0 \\
0 & b & 0 & 0
\end{array}\right] .
$$

Furthermore, we set $\Gamma_{1}:=\gamma I_{4}$, so that the $\mathrm{I}(1, r)$ conditions are met, provided $|\gamma|<1$. In the following, results are reported for $\gamma \in\{0.0,0.1,0.2,0.3,0.5,0.8,0.9\}$. Notice that the system in (5.1) approaches the $\mathrm{I}(2)$ boundary as $\gamma$ approaches unity.

In the context of (5.1) we consider for the individual components of $\varepsilon_{t}$ the univariate innovation processes and parameter configurations used in CRT2013 which correspond to those used in section 4 of Gonçalves and Kilian (2004) and in section 5 of CRT2010b, to which the reader is referred for further discussion. These are as follows:

- Case A. $\varepsilon_{i t}, i=1, \ldots, p$, is an independent sequence of $N(0,1)$ variates.

- Case B. $\varepsilon_{i t}, i=1, \ldots, p$, is an independent sequence of Student $t(\nu)$ (normalised to unit variance) variates. Results are reported for $\nu=5$.

- Case C. $\varepsilon_{i t}$ is a standard $G A R C H(1,1)$ process driven by standard normal innovations of the form $\varepsilon_{i t}=h_{i t}^{1 / 2} v_{i t}, i=1, \ldots, p$, where $v_{i t}$ is i.i.d. $N(0,1)$, independent across $i$, and $h_{i t}=$ $\omega+d_{0} \varepsilon_{i t-1}^{2}+d_{1} h_{i t-1}, t=0, \ldots, T$. Results are reported for $d_{0}=0.05$ and $d_{1}=0.94$.

- Case D. $\varepsilon_{i t}$ is the first-order AR stochastic volatility [SV] model: $\varepsilon_{i t}=v_{i t} \exp \left(h_{i t}\right), h_{i t}=$ $\lambda h_{i t-1}+0.5 \xi_{i t}$, with $\left(\xi_{i t}, v_{i t}\right)^{\prime} \sim$ i.i.d. $N\left(0, \operatorname{diag}\left(\sigma_{\xi}^{2}, 1\right)\right)$, independent across $i=1, \ldots, p$. Results are reported for $\lambda=0.951, \sigma_{\xi}=0.314$.

- Case E. $\varepsilon_{i t}$ is a non-stationary, heteroskedastic independent sequence of $N\left(0, \sigma_{i t}^{2}\right)$ variates, where $\sigma_{i t}^{2}=1$ for $t \leq\lfloor T \tau\rfloor$ and $\sigma_{i t}^{2}=\kappa$ for $t>\lfloor T \tau\rfloor$, all $i=1, \ldots, p$. Results are reported for $\tau=2 / 3$ and $\kappa=3$ (late positive variance shift).

Both Cases A and B satisfy Assumption $\mathcal{V}$ (i.i.d. shocks). Under Case C, for the chosen parameter configuration, $\varepsilon_{t}$ is globally stationary with finite $4^{\text {th }}$ order moments and, thus, satisfies Assumption $\mathcal{V}$ '. Similarly, the SV model of Case D is strictly stationary with bounded fourth order moments; see Carrasco and Chen (2002), and, hence, also satisfies Assumption V'. Finally, Case E involves a single, permanent shift in the innovation variance, thus leading to error sequences which are globally heteroskedastic and satisfy Assumption V".

All experiments are run over 10,000 Monte Carlo replications. For the bootstrap tests, any replications in which the algorithm generates explosive samples are discarded and the experiment continues until 10,000 valid replications are obtained. For each bootstrap procedure we report the frequency with which such violations occur 5 The number of replications used in the wild bootstrap algorithms is set

\footnotetext{
${ }^{5}$ As shown in CRT2012 and CRT2013 the frequency of times with which the bootstrap algorithm fails to generate I(1)
} 
to 399. The PLR and bootstrap PLR tests were all conducted at the nominal 0.05 significance level ${ }^{6}$ Throughout the Tables of results which follow the frequencies with which the various procedures select the true co-integration rank are highlighted in bold text.

We first consider the case of no co-integration in Section 5.1, then the case of a single co-integration vector in Section 5.2 and finally two co-integration relations in Section 5.3. In each of these three cases a restricted constant was fitted for the deterministic component. The corresponding results for the restricted trend will also be discussed at the end of this section.7

\subsection{The No Co-integration Case $\left(r_{0}=0\right)$}

We first report results for the case of no co-integration $\left(r_{0}=0\right)$ which obtains by setting $a=b=0$ in the long-run parameter vector $\alpha$ in (5.2). In this case (5.1) reduces to the VAR(1) in first differences, $\Delta X_{t}=\Gamma_{1} \Delta X_{t-1}+\varepsilon_{t}, t=1, \ldots, T$.

We first consider the frequencies of co-integration rank determination for the sequential procedures of the different methods for Case A (i.i.d. Gaussian shocks) and Case B (i.i.d. $t(5)$ shocks), which are reported in Tables 1.1 and 1.2, respectively.

\section{[ TABLES 1.1-1.2 ABOUT HERE ]}

Since all the tests are run at the (asymptotic) 5\% significance level, both the sequential procedures based on the standard asymptotic PLR and the wild bootstrap should (in the limit) select $r=0$ with probability $95 \%$ and $r>0$ with probability 5\%. The results in Tables 1.1 and 1.2 show that the standard sequential procedure based on $Q_{r, T}$ can display very poor performance relative to this asymptotic benchmark, in particular when $\gamma=0.8$ or 0.9 , or where the sample size is not very large. For example, when $\gamma=0.9$ and $T=50$ and with Gaussian shocks the standard procedure over-fits the co-integration rank about $95 \%$ of the time, selecting a rank of either 3 or 4 over $40 \%$ of the time, even though the true rank is zero. For $T=400$ the behaviour of the standard procedure in this example is much improved although it still overestimates the co-integration rank in about a quarter of cases. In contrast, and in line with the results in CRT2013, the analogous sequential procedure based on the bootstrap $Q_{r, T}^{* w}$ tests is seen to deliver far superior finite sample performance throughout. In almost all cases the empirical frequency with which it selects the true co-integration rank lies very close to 95\%. A comparison between the results in Tables 1.1 and 1.2, suggests that both the asymptotic and bootstrap sequential procedures are little affected by whether the shocks are Gaussian or $t(5)$ distributed.

samples, thus violating the root check condition denoted in step (ii), is quite low. The percentages reported in columns labelled RC in Tables 1.1-1.5, 2.1-2.5, and 3.1-3.5 confirm their results. A notable number of violations are seen to occur when volatility is persistent (Cases D and E) and the sample size is very small $(T=50)$, but the frequency of such failures decreases rapidly as the sample size increases.

${ }^{6}$ All the simulations reported in the paper were programmed using MATLAB. Our MATLAB programs are available on request.

${ }^{7}$ For the standard PLR tests the asymptotic critical values used are taken from Table 15.2 of Johansen (1996). 
Consider next the results for the information criteria in Tables 1.1 and 1.2. In line with results reported in previous studies (see, e.g., Kapetanios, 2004; Wang and Bessler, 2005) the AIC-based procedure is shown to be completely unreliable. Even in the simplest case where $\gamma=0$ and the shocks are Gaussian, the AIC-based procedure detects the true co-integration rank, $r_{0}=0$, around $7 \%$ of the time for $T=50$, increasing to only $20 \%$ for $T=4008$ The performance of the AIC-based procedure is even worse when $\gamma \neq 0$. In contrast, the BIC-based procedure generally performs well. Indeed BICbased procedure selects $r=0$ at least $98.9 \%$ of the time when $T=200$ and reaches (or approaches) $100 \%$ when $T=400$, for all values of $\gamma$ considered and regardless of whether the shocks are Gaussian or $t(5)$ distributed. However, the BIC-based procedure does display a significant tendency to overestimate the co-integration rank when the sample size is small $(T \leq 100)$, with this effect becoming stronger, others things being equal, as $\gamma$ increases. The HQC-based procedure is dominated by the BIC procedure throughout the results in Tables 1.1-1.2 but is clearly preferable to the AIC procedure.

We now turn to a discussion of the results for the two (stationary) conditionally heteroskedastic processes specified in Cases $\mathrm{C}$ (independent stationary $\operatorname{GARCH}(1,1)$ processes) and D (stationary autoregressive SV processes) above. For $r_{0}=0$, results for the different methods are reported in Tables 1.3 (Case C) and 1.4 (Case D).

\section{[ TABLES 1.3-1.5 ABOUT HERE ]}

Comparing the results in Table 1.3 with those in Tables 1.1 and 1.2, it is seen that the results for each of the various procedures under stationary $\operatorname{GARCH}(1,1)$ errors are very similar to those obtained under i.i.d. Gaussian and $t(5)$ errors (Cases A and B). However, and in line with the simulation results reported in CRT2010b and CRT2013, a comparison of the results in Tables 1.4 with those in Tables 1.1 and 1.2 shows a significant decline in the behaviour of the standard sequential procedure under the autoregressive SV model 9 Of the two stationary conditionally heteroskedastic innovation process considered, the autoregressive SV (Case D) model clearly highlights the benefits of using a sequential procedure based on the wild bootstrap tests rather than the standard PLR tests. Indeed, throughout Table 1.4 the empirical frequencies with which $r=0$ is selected by the sequential procedure based on the wild bootstrap $Q_{r, T}^{* w}$ tests remain close to $95 \%$. Like the standard sequential procedure, the finite sample performance of the information criteria-based methods is also affected by the presence of $\mathrm{SV}$ in the shocks. For $T=50$, the most reliable of these, the BIC-based procedure, selects the true co-integration rank $74.8 \%$ of the time when $\gamma=0$ and this frequency drops to $35.4 \%$ when $\gamma=0.9$. As with the previous cases considered, the performance of the AIC-based procedure is highly unsatisfactory, while the HQC-based procedure shows markedly worse performance than in the

\footnotetext{
${ }^{8}$ Notice, of course, that the tendency of the AIC-based procedure to overestimate the co-integration rank will necessarily appear most pronounced in the current scenario where the true co-integration rank is zero, as compared say to a case where the true rank was closer to the VAR dimension, $p$.

${ }^{9}$ As explained in CRT2013, the chosen parameter configuration for Case D implies relatively strong serial dependence in the conditional variance of the innovations, which has a considerable impact on the finite sample performance of asymptotic PLR test.
} 
previous cases considered such that, for example, it even has difficulty in identifying $r_{0}=0$ even when $T=400$.

We next turn to a discussion of the results for the non-stationary heteroskedastic model in Case E. The results are reported in Table 1.5. Under a single, permanent shift in volatility the sequential procedure based on the asymptotic test is seen to be extremely unreliable: even in the case where $\gamma=0$ and $T=400$, the frequency of determining the true co-integration rank remains below $60 \%$. Conversely, the procedure based on the wild bootstrap remains reliable at least in the larger sample sizes reported. However, when $T=50$, the wild bootstrap-based sequential procedure shows a slight tendency to over-estimate the co-integration rank, although this is quickly ameliorated as the sample size increases; for example, when $\gamma=0.9(\gamma=0)$, the frequency of detecting $r_{0}=0$ when $T=50$ is $86.9 \%$ (90.0\%), increasing to $94.7 \%$ (94.5\%) for $T=400$. The finite sample performances of all of the information criterion-based procedures are clearly affected by the presence of a single volatility break. The BIC-based procedure remains the most reliable of the information-based methods but, nonetheless, it does badly over-estimate the co-integration rank for sample sizes smaller than $T=200$. However, for samples of size $T=200$ and larger the BIC-based procedure tends to over-estimate the co-integration rank less frequently than the wild bootstrap approach. In contrast the HQC-based and, in particular, AIC-based procedures appear very unreliable in the presence of unconditional heteroskedasticity; indeed, even for $\gamma=0$ and $T=400$, the frequency of identifying $r_{0}=0$ is $4.3 \%$ and $64.1 \%$ for AIC and HQC, respectively.

Overall then, our results for the non-co-integrated case, suggest that the sequential procedure based on the wild bootstrap algorithm provides reliable results for all the conditional and unconditional heteroskedastic processes considered. Among the information criteria, the BIC-based procedure performs well in many cases analysed, particular so when the sample size is large where it often outperforms the wild bootstrap-based procedure. In contrast the HQC-based and, in particular, the AIC-based procedures do not appear reliable in practice, most notably so where unconditional heteroskedasticity is present.

\subsection{The Co-integrated Case with $r_{0}=1$}

We now consider the case of a single co-integration vector. In this case, as in CRT2012 and CRT2013 we consider the $\operatorname{VAR}(2)$ in (5.1) where we set $a=-0.4$ and $b=0$ in the long-run parameter vector $\alpha$ in 5.2), thus obtaining $\beta^{\prime}=(1,0,0,0)$ and $\alpha^{\prime}=(-0.4,0,0,0)$. For these parameter combinations, $X_{t}$ is $\mathrm{I}(1)$ with co-integrating rank $r_{0}=1$.

\section{[ TABLES 2.1-2.2 ABOUT HERE ]}

The results for i.i.d. shocks, analogous to those reported in Tables 1.1 and 1.2, are reported in Tables 2.1 (Gaussian shocks) and $2.2(t(5)$ shocks). These results again confirm the simulation findings of CRT2012 that the standard sequential procedure again performs poorly in the $r_{0}=1$ case under both Gaussian and $t(5)$ shocks. In contrast, the sequential procedure based on the wild bootstrap again 
performs reasonably well selecting the true co-integrating rank close to the nominal $95 \%$ frequency in most cases when $T \geq 200$, especially so where $\gamma$ is large. However, when the sample size and the value of parameter $\gamma$ are small, namely $T \leq 100$ and $\gamma \leq 0.3$, it is seen that the bootstrap sequential procedure based on $Q_{r, T}^{* w}$ displays a tendency to under-estimate the co-integration rank selecting $r=0$, rather than the true rank of one, at least $50 \%$ of the time. As regards the information criterion-based methods, the results in Tables 2.1 and 2.2 show that the BIC-based approach correctly identifies the true co-integration rank, $r_{0}=1$, almost $100 \%$ of the time when $T=400$, under both Gaussian and $t(5)$ shocks. However, as was seen for the $r_{0}=0$ case, its performance is affected by the value of $\gamma$; specifically its performance is significantly better when $\gamma$ is large $(\gamma \geq 0.5)$, although this property in itself is largely an artefact of the tendency of the BIC-based approach to over-estimate the cointegration rank in such cases in the $r_{0}=0$ case seen in Tables 1.1 and 1.2 . When $\gamma \leq 0.3$ and the sample size is small, $T \leq 100$, the HQC-based procedure is preferable to the BIC-based procedure for both Gaussian and $t(5)$ shocks, although again this is largely an artefact of the greater tendency of the former to over-estimate the co-integration rank in such cases when $r_{0}=0$. The results in Tables 2.1-2.2 again highlight the inadequacy of the AIC-based procedure.

We now turn to the results for the two (stationary) conditionally heteroskedastic processes, Cases $\mathrm{C}$ and D, which are reported in Tables 2.3 and 2.4, respectively.

\section{[ TABLES 2.3-2.5 ABOUT HERE ]}

As with the corresponding results for $r_{0}=0$, reported in Tables 1.1-1.4, the performances of all of co-integration procedures considered are little affected by the form of conditional heteroskedasticity specified under Case C, relative to the case of i.i.d. shocks, but are significantly affected under the autoregressive SV model specified in Case D. For example, while the standard sequential procedure based on the asymptotic PLR tests correctly estimates the true rank $94 \%$ of the time when $\gamma=0$ under i.i.d. shocks when $T=400$ this reduces to only $80 \%$ under SV. In contrast, the wild bootstrap-based sequential procedure again works well, especially when $T=400$ and $\gamma$ is large. Among the information criterion-based procedures, the BIC-based approach appears overall to be the most reliable under SV, except where both $\gamma$ and $T$ are small in which case the HQC-based approach is preferable. Indeed, except for the case where $\gamma=0.9$, the BIC-based procedure outperforms the wild bootstrap-based procedure in terms of its frequency of selecting the true co-integration rank, although once again this is largely attributable to the tendency shown by the former to over-estimate the rank when $r_{0}=0$; see Table 1.4.

We conclude this section by turning to the results reported in Table 2.5 for the non-stationary heteroskedastic Case E. As was previously observed in Section 5.1 for the non-co-integrated model, the finite sample performances of all of the procedures considered are strongly affected by the presence of a single, permanent shift in the innovation variance. In particular, even for $T=400$, the standard sequential procedure correctly estimates the true co-integration rank only around $75 \%$ of the time when $\gamma \leq 0.5$, with this frequency being even lower when $\gamma \geq 0.8$. In contrast, for $T=400$, the wild 
bootstrap-based sequential procedure correctly estimates the co-integration rank to be one at close to the nominal 95\% level, confirming its superiority over the standard procedure based on $Q_{r, T}$ in large samples under non-stationary volatility. It should be stressed, however, that for small values of $\gamma$ and $T$, the wild bootstrap-based procedure tends to underestimate the co-integration rank; for example, when $\gamma=0.3$ and $T=100$, it determines the true co-integration rank only $40.1 \%$ of the time, while it selects $r=0$ with frequency 54.3\%. Overall, for these combinations of $\gamma$ and $T$, the standard Johansen procedure outperforms the corresponding wild bootstrap procedure, although again this is an artefact of the relative size properties of these tests; cf. Table 1.5. The ability of the information criterion-based approaches to detect the true co-integration rank also deteriorates under Case E. For example, in the presence of a single break in volatility and where $\gamma=0.5$, the BIC-based procedure estimates the rank to be one around $48 \%$ when $T=50$, increasing to around $74 \%(95 \%)$ when $T=100(T=200)$, and above $98 \%$ when $T=400$. Under Case $\mathrm{E}$ the BIC-based procedures again significantly outperforms the HQC- and AIC-based procedures. Moreover, as was seen under Case D, the BIC-based procedure again significantly outperforms (although again the behaviour of these procedures under $r_{0}=0$ partly explains these differences) the wild bootstrap-based procedure except when $\gamma$ is large $(\gamma \geq 0.8)$ where this can be reversed.

Overall, when there is a single co-integration relation in the DGP, the sequential procedure based on the wild bootstrap algorithm displays very good performance for all the forms of heteroskedasticity considered when the sample size is large. Among the information criteria methods, the BIC-based procedure delivers the best performance with different heteroskedastic innovation processes having only limited impact on its efficacy.

\subsection{The Case of Two Co-integration Relations, $r_{0}=2$}

The case of two co-integration relations is obtained by setting $a=b=-0.4$ in the long-run vector $\alpha$ in $(5.2)$ of the $\operatorname{VAR}(2)$ model in (5.1). In this case, $X_{t}$ is I(1) with co-integrating rank $r_{0}=2 \sqrt{10}$ The associated results for the five configurations of the innovation process $\varepsilon_{t}$ specified in Cases A-E are reported in Tables 3.1-3.5.

\section{[ TABLES 3.1 - 3.5 ABOUT HERE ]}

From the results in Tables 3.1-3.5, it can be noted that, when the sample size is large, the performance of the standard sequential procedure again deteriorates when moving from i.i.d. and GARCH innovations (Cases A, B, and C) to SV and unconditionally heteroskedastic shocks (Cases D and E). However, perhaps surprisingly, for the smaller values of $T$ considered, the sequential procedure based on $Q_{r, T}$ tends to determine the true co-integration rank, $r_{0}=2$, more frequently under Cases $\mathrm{D}$ and $\mathrm{E}$ than it does under Cases A-C. This is particularly evident when $\gamma$ is small. Conversely, the bootstrap-based procedure shows excellent overall performance when the sample is large; for example, when $T=400$, the true rank is selected close to the nominal frequency of $95 \%$ for all the cases

\footnotetext{
${ }^{10}$ The case of $r_{0}=2$ is not analysed in CRT2012 or CRT2013.
} 
considered. However, when $T=50$, the standard sequential procedure outperforms the corresponding bootstrap procedure, regardless of the value of the autoregressive parameter $\gamma$ and the form of heteroskedasticity considered (although again we note that this is largely an artefact of the relative sizes of the tests involved in the bootstrap and standard procedures). This pattern is also observed when $T=100$ and $\gamma \leq 0.5$. As was already noted in Sections 5.1 and 5.2, the BIC-based approach clearly outperforms the corresponding HQC- and AIC-based approaches when the sample size is large (i.e., $T \geq 200$ ), for all values of $\gamma$ considered. Moreover, it can be observed from the results in Tables 3.1-3.5 that the small sample performance of the BIC-based approach tends to improve as $\gamma$ increases. Where the sample size is small $(T \leq 100)$ and when $\gamma \leq 0.5$ the HQC-based procedure outperforms the BIC-based procedure. The AIC-based procedure again tends to over-estimation rank; this is less pronounced than was the case for $r_{0}=1$ and $r_{0}=2$ for the reasons discussed in footnote 8 .

Thus far we have only discussed the case of a restricted constant. We also repeated the foregoing experiments for the restricted trend case. We do not report these results here but they are available from the authors on request. Overall, and as might be expected, the results for the restricted trend case are qualitatively similar to the corresponding restricted constant results, but with an overall deterioration observed in the performance of all of the procedures the more so the smaller the sample size, other things being equal. An interesting exception to this general pattern is seen for the BICbased approach whose performance in the restricted trend case is, if anything, improved rather than worsened relative to the restricted constant case when $r_{0}=1$ or 2 and where the value of $\gamma$ is small.

\section{Conclusions}

In this paper we have analysed the performance of various methods for determining the co-integration rank in heteroskedastic VAR models. We have compared the efficacy to do so of the standard sequential procedure based on the asymptotic PLR test of Johansen (1996), the wild bootstrap analogue of this procedure due to Cavaliere, Rahbek and Taylor (2010a, 2010b, 2013), and methods based on widely used information criteria (specifically, AIC, HQC, and BIC). For all these methods, we have evaluated their asymptotic properties in the presence of heteroskedasticity (conditional or unconditional) in the shocks of a quite general and unknown form, complementing the results given in Cavaliere, Rahbek and Taylor (2013), and conducted a Monte Carlo simulation study into their relative performance in finite samples. Our numerical results have suggested that the BIC-based procedure and the wild bootstrap sequential test procedure are the most reliable of the available methods for determining the co-integration rank, at least for the simulation DGPs considered. The BIC-based method was competitive and even, in certain cases, displayed superior performance to the wild bootstrap procedure for larger sample sizes (noting that, in contrast to the BIC-based procedure, the probability of selecting the true rank for the bootstrap sequential procedure is bounded by the choice of nominal significance, even asymptotically). As such, the BIC-based and bootstrap-based sequential methods usefully complement each other in practice.

All of the methods we have discussed in this paper are based on the assumption that the value of 
the lag length parameter $k$ in 2.1 is finite and known, or at least has been pre-determined by the practitioner. An interesting direction for further research is therefore to develop implementations of the procedures outlined in this paper that simultaneously estimate the co-integration rank and the lag parameter $k$ in the presence of heteroskedastic shocks, and to compare these approaches with the semiparametric and non-parametric information criterion co-integration rank determination procedures of Cheng and Phillips (2009) and Poskitt (2000), neither of which require this parameter to be known or finite. This is currently under investigation by the authors.

\section{References}

Akaike, H. (1974): A new look at the statistical model identification, IEEE Transactions on Automatic Control 19, 716-723.

Aznar, A. and M. Salvador (2002): Selecting the rank of the cointegration space and the form of the intercept using an information criterion, Econometric Theory 18, 926-947.

Baltagi, B.H. and Z. Wang (2007): Testing for cointegrating rank via model selection: evidence from 165 data sets, Empirical Economics 33, 41-49.

Carrasco, M. and X. Chen (2002): Mixing and moment properties of various Garch and stochastic volatility models, Econometric Reviews 18, 17-39.

Cavaliere, G., A. Rahbek and A.M.R. Taylor (2010a): Co-integration rank testing under conditional heteroskedasticity, Econometric Theory 26, 1719-1760.

Cavaliere, G., A. Rahbek and A.M.R. Taylor (2010b): Testing for co-integration in vector autoregressions with non-stationary volatility, Journal of Econometrics 158, 7-24.

Cavaliere, G., A. Rahbek and A.M.R. Taylor (2012): Bootstrap determination of the co-integration rank in VAR models, Econometrica 80, 1721-1740.

Cavaliere, G., A. Rahbek and A.M.R. Taylor (2013): Bootstrap determination of the co-integration rank in heteroskedastic VAR models, forthcoming in Econometric Reviews.

Cheng, X. and P.C.B. Phillips (2009): Semiparametric cointegrating rank selection, Econometrics Journal 12, 83-104.

Cheng, X. and P.C.B. Phillips (2012): Cointegrating rank selection in models with time-varying variance, Journal of Econometrics 169, 155-165.

Davidson, J. (1994): Stochastic limit theory, Oxford: Oxford University Press.

Gonçalves, S. and L. Kilian (2004): Bootstrapping autoregressions with conditional heteroskedasticity of unknown form, Journal of Econometrics 123, 89-120. 
Hannan, E.J. and B.G. Quinn (1979): The determination of the order of an autoregression, Journal of the Royal Statistical Society, Series B 41, 190-195.

Johansen, S. (1988): Statistical analysis of cointegration vectors, Journal of Economic Dynamics and Control 12, 231-254.

Johansen, S. (1991): Estimation and hypothesis testing of cointegration vectors in Gaussian vector autoregressive models, Econometrica 59, 1551-1580.

Johansen, S. (1996): Likelihood-based inference in cointegrated vector autoregressive models, Oxford: Oxford University Press.

Johansen, S. (2002): A small sample correction of the test for cointegrating rank in the vector autoregressive model, Econometrica 70, 1929-1961.

Kapetanios, G. (2000): Information criteria, model selection uncertainty and the determination of cointegration rank, NIESR Discussion Papers 166, National Institute of Economic and Social Research.

Kapetanios, G. (2004): The asymptotic distribution of the cointegration rank estimator under the Akaike information criterion, Econometric Theory 20, 735-742.

Kascha, C. and C. Trenkler (2011): Bootstrapping the likelihood ratio cointegration test in error correction models with unknown lag order, Computational Statistics and Data Analysis 55, $1008-1017$.

Lee, T.-H. and Y. Tse (1996): Cointegration tests with conditional heteroskedasticity, Journal of Econometrics 73, 401-410.

McConnell, M.M. and G. Perez Quiros (2000): Output fluctuations in the United States: what has changed since the early 1980s?, American Economic Review 90, 1464-1476.

Nielsen, B. (2006): Order determination in general vector autoregressions, IMS Lecture Notes Monograph Series 52, 93-112.

Paulsen, J. (1984): Order determination of multivariate autoregressive time series with unit roots, Journal of Time Series Analysis 5, 115-127.

Poskitt, D.S. (2000): Strongly consistent determination of cointegrating rank via canonical correlations, Journal of Business and Economic Statistics 18, 71-90.

Rissanen, J. (1978): Modeling by shortest data description, Automatica 14, 465-471.

Schwarz, G. (1978): Estimating the dimension of a model, Annals of Statistics 6, 461-464.

Sensier, M., and D. van Dijk (2004): Testing for volatility changes in U.S. macroeconomic time series, Review of Economics and Statistics 86, 833-839. 
van Dijk, D., D.R. Osborn and M. Sensier (2002): Changes in variability of the business cycle in the G7 countries. Erasmus University Rotterdam. Econometric Institute Report EI 2002-28.

Wang, Z. and D.A. Bessler (2005): A Monte Carlo study on the selection of cointegration rank using information criteria, Econometric Theory 21, 593-620.

\section{A Appendix}

Proof of Theorem 1: The result under Assumption $\mathcal{V}$ is proved in Johansen (1996), while the results under Assumptions $\mathcal{V}^{\prime}$ and $\mathcal{V}$ ", are established in CRT2010a.

Proof of Theorem 2: Part (i) is established in Corollary 1 of CRT2012 and the part (ii) is established in Corollary 1 of CRT2013.

Proof of Theorem 3. The outline of the proof mimics the proof of Aznar and Salvador (2002, proof of Theorem 4.1), applying results here from CRT2010a under conditional heteroskedastic innovations, viz, under Assumption $\mathcal{V}$ ' (see Remark 3.4 in CRT2010a), and for the case of unconditional heteroskedastic innovations (Assumption $\mathcal{V}$ ") by CRT2010b (see their Remark 3.2). Specifically, for any $r \neq r_{0}$, we have that

$$
\mathrm{IC}(r)-\mathrm{IC}\left(r_{0}\right)= \begin{cases}T \sum_{i=r_{0}+1}^{r} \log \left(1-\hat{\lambda}_{i}\right)+c_{T}\left(2 p-r-r_{0}\right)\left(r-r_{0}\right) & \text { for } r>r_{0} \\ -T \sum_{i=r+1}^{r_{0}} \log \left(1-\hat{\lambda}_{i}\right)+c_{T}\left(2 p-r-r_{0}\right)\left(r-r_{0}\right) & \text { for } r<r_{0}\end{cases}
$$

When $r>r_{0}$, to show that $\operatorname{Prob}\left(\mathrm{IC}(r)-\mathrm{IC}\left(r_{0}\right)>0\right) \rightarrow 1$ as $T \rightarrow \infty$, with $c_{T} \rightarrow \infty$, consider

$$
\mathrm{IC}(r)-\operatorname{IC}\left(r_{0}\right)=T \sum_{i=r_{0}+1}^{r} \log \left(1-\hat{\lambda}_{i}\right)+c_{T}\left(2 p-r-r_{0}\right)\left(r-r_{0}\right) .
$$

As in Johansen (1996, p. 160), and using here Remarks 3.4 and 3.2 of CRT2010a and CRT2010b, respectively, the first term in A.1 satisfies

$$
T \sum_{i=r_{0}+1}^{r} \log \left(1-\hat{\lambda}_{i}\right)=O_{p}(1), \text { for all } i=r_{0}+1, \ldots, r .
$$

The second term of (A.1) is positive and dominates the first term if $c_{T} \rightarrow \infty$. Thus, $r>r_{0}$ will never be selected if $c_{T}$ in the penalty term diverges as $T \rightarrow \infty$.

When $r<r_{0}$ (and $r_{0} \neq 0$ ),

$$
\mathrm{IC}(r)-\mathrm{IC}\left(r_{0}\right)=-T \sum_{i=r+1}^{r_{0}} \log \left(1-\hat{\lambda}_{i}\right)+c_{T}\left(2 p-r-r_{0}\right)\left(r-r_{0}\right) .
$$

The right side of A.2 involves a subset of the largest $r_{0}$ eigenvalues $\hat{\lambda}_{i}$, for $i=1, \ldots, r_{0}$. Under either Assumption $\mathcal{V}, \mathcal{V}$, or $\mathcal{V}$ ", as in Lemma 12.1 in Johansen (1996), these $r_{0}$ sample eigenvalues converge to the $r_{0}$ solutions of $\left|\lambda \Sigma_{\beta \beta}-\Sigma_{\beta 0} \Sigma_{00}^{-1} \Sigma_{0 \beta}\right|=0$, where $\Sigma_{\beta \beta}, \Sigma_{\beta 0}:=\Sigma_{0 \beta}^{\prime}$, and $\Sigma_{00}$ are defined in CRT2010a (pp. 1753-1754) and CRT2010b (p. 22), these being all positive. Consequently, 
$-\sum_{i=r+1}^{r_{0}} \log \left(1-\hat{\lambda}_{i}\right)$ is strictly positive in the limit and therefore the first term on the right side in A.2 diverges to $+\infty$ as $T \rightarrow \infty$. Conversely, since $\left(r-r_{0}\right)<0$, the second term in A.2 diverges to $-\infty$ as $c_{T} \rightarrow \infty$ and $T \rightarrow \infty$. Thus, if $c_{T} / T \rightarrow 0$ as $T \rightarrow \infty$ then the first term in A.2 dominates and $\operatorname{Prob}\left(\mathrm{IC}(r)>\operatorname{IC}\left(r_{0}\right)\right) \rightarrow 1$. Hence, $r<r_{0}$ will never be selected if $c_{T}$ in the penalty term is of order $o(T)$. 
TABLE 1.1: Sequential procedures for determining the co-integration rank. $\operatorname{VAR}(2)$ model with rank $r_{0}=0$, i.i.d. Gaussian errors [Case A]

\begin{tabular}{|c|c|c|c|c|c|c|c|c|c|c|c|c|c|c|c|c|c|c|c|c|c|c|}
\hline \multirow[b]{2}{*}{$\gamma$} & \multirow[b]{2}{*}{$T$} & \multicolumn{4}{|c|}{ Johansen } & \multicolumn{5}{|c|}{ Bootstrap } & \multicolumn{4}{|c|}{ AIC } & \multicolumn{4}{|c|}{$\mathrm{HQC}$} & \multicolumn{4}{|c|}{ BIC } \\
\hline & & $r=0$ & 1 & 2 & 3,4 & $r=0$ & 1 & 2 & 3,4 & $\mathrm{RC}$ & $r=0$ & 1 & 2 & 3,4 & $r=0$ & 1 & 2 & 3,4 & $r=0$ & 1 & 2 & 3,4 \\
\hline \multirow[t]{4}{*}{0.0} & 50 & 80.1 & 16.9 & 2.6 & 0.4 & 96.5 & 3.1 & 0.3 & 0.0 & 0.0 & 7.3 & 25.4 & 29.1 & 38.1 & 41.7 & 38.9 & 13.1 & 6.3 & 89.4 & 9.9 & 0.7 & 0.1 \\
\hline & 100 & 89.4 & 9.4 & 1.0 & 0.1 & 95.5 & 4.1 & 0.3 & 0.1 & 0.0 & 13.6 & 34.1 & 26.8 & 25.6 & 74.3 & 22.2 & 2.9 & 0.7 & 98.9 & 1.1 & 0.0 & 0.0 \\
\hline & 200 & 92.3 & 7.0 & 0.6 & 0.2 & 95.3 & 4.4 & 0.3 & 0.1 & 0.0 & 18.5 & 35.8 & 24.5 & 21.3 & 88.2 & 10.8 & 0.9 & 0.1 & 99.9 & 0.1 & 0.0 & 0.0 \\
\hline & 400 & 93.5 & 5.9 & 0.6 & 0.1 & 95.1 & 4.4 & 0.5 & 0.1 & 0.0 & 20.4 & 36.9 & 24.3 & 18.4 & 94.2 & 5.5 & 0.3 & 0.0 & 100.0 & 0.0 & 0.0 & 0.0 \\
\hline \multirow[t]{4}{*}{0.1} & 50 & 77.6 & 18.9 & 3.0 & 0.6 & 96.9 & 2.7 & 0.4 & 0.0 & 0.0 & 6.3 & 24.1 & 29.1 & 40.5 & 39.4 & 38.7 & 14.6 & 7.3 & 88.5 & 10.7 & 0.8 & 0.1 \\
\hline & 100 & 88.6 & 10.2 & 1.0 & 0.2 & 96.1 & 3.5 & 0.4 & 0.0 & 0.0 & 12.9 & 33.1 & 27.3 & 26.7 & 73.1 & 23.1 & 3.1 & 0.7 & 98.9 & 1.1 & 0.0 & 0.0 \\
\hline & 200 & 92.0 & 7.2 & 0.6 & 0.2 & 95.5 & 4.1 & 0.3 & 0.1 & 0.0 & 18.0 & 35.7 & 24.6 & 21.7 & 87.9 & 11.1 & 0.9 & 0.1 & 99.9 & 0.1 & 0.0 & 0.0 \\
\hline & 400 & 93.3 & 5.9 & 0.7 & 0.1 & 95.4 & 4.2 & 0.4 & 0.1 & 0.0 & 20.3 & 36.7 & 24.3 & 18.7 & 94.0 & 5.7 & 0.3 & 0.0 & 100.0 & 0.0 & 0.0 & 0.0 \\
\hline \multirow[t]{4}{*}{0.2} & 50 & 74.4 & 21.5 & 3.3 & 0.8 & 96.7 & 2.9 & 0.4 & 0.1 & 0.0 & 5.2 & 22.0 & 28.9 & 43.9 & 36.3 & 39.1 & 16.1 & 8.5 & 87.3 & 11.6 & 1.0 & 0.1 \\
\hline & 100 & 87.4 & 11.3 & 1.1 & 0.2 & 95.9 & 3.7 & 0.4 & 0.0 & 0.0 & 12.1 & 32.1 & 27.7 & 28.1 & 72.0 & 24.0 & 3.2 & 0.9 & 98.7 & 1.3 & 0.0 & 0.0 \\
\hline & 200 & 91.6 & 7.6 & 0.6 & 0.2 & 95.3 & 4.2 & 0.4 & 0.1 & 0.0 & 17.6 & 35.4 & 24.7 & 22.3 & 87.4 & 11.4 & 1.0 & 0.1 & 99.9 & 0.1 & 0.0 & 0.0 \\
\hline & 400 & 93.0 & 6.3 & 0.7 & 0.1 & 95.6 & 4.1 & 0.2 & 0.0 & 0.0 & 20.1 & 36.5 & 24.4 & 19.1 & 94.0 & 5.7 & 0.3 & 0.0 & 100.0 & 0.0 & 0.0 & 0.0 \\
\hline \multirow[t]{4}{*}{0.3} & 50 & 70.4 & 24.3 & 4.2 & 1.0 & 96.8 & 2.9 & 0.2 & 0.1 & 0.0 & 4.1 & 19.9 & 28.1 & 47.9 & 33.1 & 39.0 & 17.8 & 10.1 & 85.8 & 12.8 & 1.2 & 0.2 \\
\hline & 100 & 86.2 & 12.2 & 1.4 & 0.3 & 95.7 & 3.9 & 0.4 & 0.1 & 0.0 & 10.9 & 31.1 & 28.1 & 29.9 & 70.2 & 25.1 & 3.7 & 1.0 & 98.6 & 1.4 & 0.0 & 0.0 \\
\hline & 200 & 91.0 & 8.1 & 0.7 & 0.2 & 95.8 & 3.8 & 0.4 & 0.0 & 0.0 & 17.1 & 34.9 & 25.1 & 22.9 & 86.8 & 12.1 & 1.0 & 0.2 & 99.9 & 0.1 & 0.0 & 0.0 \\
\hline & 400 & 92.8 & 6.4 & 0.8 & 0.1 & 95.5 & 4.1 & 0.3 & 0.1 & 0.0 & 19.7 & 36.4 & 24.6 & 19.4 & 93.7 & 5.9 & 0.3 & 0.0 & 100.0 & 0.0 & 0.0 & 0.0 \\
\hline \multirow[t]{4}{*}{0.5} & 50 & 57.5 & 32.4 & 7.9 & 2.3 & 95.8 & 3.8 & 0.4 & 0.1 & 0.0 & 2.0 & 12.8 & 24.9 & 60.3 & 24.3 & 36.7 & 22.0 & 17.1 & 80.2 & 17.4 & 2.0 & 0.4 \\
\hline & 100 & 80.7 & 16.6 & 2.2 & 0.5 & 95.7 & 4.0 & 0.3 & 0.1 & 0.0 & 8.1 & 27.9 & 27.9 & 36.1 & 65.2 & 27.9 & 5.3 & 1.6 & 97.9 & 2.0 & 0.1 & 0.0 \\
\hline & 200 & 89.0 & 9.7 & 1.0 & 0.3 & 95.2 & 4.3 & 0.4 & 0.1 & 0.0 & 14.7 & 33.7 & 26.5 & 25.1 & 84.9 & 13.6 & 1.3 & 0.2 & 99.8 & 0.2 & 0.0 & 0.0 \\
\hline & 400 & 92.1 & 7.0 & 0.8 & 0.1 & 94.8 & 4.6 & 0.5 & 0.1 & 0.0 & 18.4 & 35.7 & 25.2 & 20.7 & 93.3 & 6.3 & 0.4 & 0.0 & 100.0 & 0.0 & 0.0 & 0.0 \\
\hline \multirow[t]{4}{*}{0.8} & 50 & 17.8 & 36.5 & 27.2 & 18.5 & 94.9 & 4.4 & 0.6 & 0.1 & 0.1 & 0.1 & 1.4 & 7.3 & 91.1 & 6.1 & 17.5 & 20.8 & 55.6 & 58.8 & 27.4 & 7.9 & 5.9 \\
\hline & 100 & 52.0 & 34.2 & 10.5 & 3.3 & 94.7 & 4.7 & 0.6 & 0.1 & 0.0 & 1.9 & 11.3 & 22.5 & 64.3 & 42.4 & 35.5 & 14.0 & 8.2 & 94.5 & 5.3 & 0.2 & 0.0 \\
\hline & 200 & 76.1 & 19.6 & 3.4 & 1.0 & 95.1 & 4.5 & 0.3 & 0.1 & 0.1 & 7.0 & 24.5 & 28.3 & 40.2 & 75.6 & 20.7 & 3.0 & 0.8 & 99.5 & 0.5 & 0.0 & 0.0 \\
\hline & 400 & 86.8 & 11.5 & 1.5 & 0.3 & 95.4 & 4.2 & 0.3 & 0.1 & 0.0 & 12.6 & 32.7 & 27.5 & 27.2 & 90.4 & 8.7 & 0.7 & 0.1 & 100.0 & 0.0 & 0.0 & 0.0 \\
\hline \multirow[t]{4}{*}{0.9} & 50 & 5.3 & 21.6 & 30.6 & 42.5 & 93.1 & 5.9 & 0.9 & 0.2 & 1.8 & 0.0 & 0.2 & 1.6 & 98.2 & 1.9 & 6.6 & 11.1 & 80.5 & 45.6 & 27.0 & 10.8 & 16.6 \\
\hline & 100 & 23.6 & 37.1 & 24.3 & 15.1 & 93.3 & 5.9 & 0.7 & 0.1 & 0.2 & 0.3 & 2.7 & 9.5 & 87.6 & 23.9 & 29.7 & 19.5 & 26.8 & 89.5 & 9.2 & 1.1 & 0.3 \\
\hline & 200 & 53.0 & 33.4 & 10.2 & 3.5 & 93.8 & 5.5 & 0.6 & 0.1 & 0.1 & 2.2 & 12.0 & 23.0 & 62.8 & 60.2 & 28.9 & 7.8 & 3.2 & 99.0 & 1.0 & 0.0 & 0.0 \\
\hline & 400 & 76.1 & 19.5 & 3.3 & 1.0 & 95.1 & 4.4 & 0.4 & 0.1 & 0.0 & 6.9 & 25.2 & 28.2 & 39.7 & 84.7 & 13.6 & 1.4 & 0.3 & 99.9 & 0.1 & 0.0 & 0.0 \\
\hline
\end{tabular}


TABLE 1.2: Sequential procedures for determining the co-integration rank. VAR(2) model with rank $r_{0}=0$, i.i.d. $t(5)$ errors [Case B]

\begin{tabular}{|c|c|c|c|c|c|c|c|c|c|c|c|c|c|c|c|c|c|c|c|c|c|c|}
\hline \multirow[b]{2}{*}{$\gamma$} & \multirow[b]{2}{*}{$T$} & \multicolumn{4}{|c|}{ Johansen } & \multicolumn{5}{|c|}{ Bootstrap } & \multicolumn{4}{|c|}{ AIC } & \multicolumn{4}{|c|}{ HQC } & \multicolumn{4}{|c|}{$\mathrm{BIC}$} \\
\hline & & $r=0$ & 1 & 2 & 3,4 & $r=0$ & 1 & 2 & 3,4 & $\mathrm{RC}$ & $r=0$ & 1 & 2 & 3,4 & $r=0$ & 1 & 2 & 3,4 & $r=0$ & 1 & 2 & 3,4 \\
\hline \multirow[t]{4}{*}{0.0} & 50 & 79.7 & 17.3 & 2.4 & 0.5 & 97.1 & 2.6 & 0.3 & 0.1 & 0.0 & 7.9 & 25.4 & 28.7 & 38.0 & 42.6 & 37.6 & 13.9 & 5.9 & 88.5 & 10.7 & 0.7 & 0.1 \\
\hline & 100 & 89.0 & 9.7 & 1.1 & 0.3 & 96.0 & 3.5 & 0.4 & 0.0 & 0.0 & 14.8 & 32.9 & 26.6 & 25.8 & 73.7 & 22.1 & 3.4 & 0.8 & 98.6 & 1.4 & 0.0 & 0.0 \\
\hline & 200 & 91.9 & 7.3 & 0.8 & 0.1 & 95.6 & 4.0 & 0.3 & 0.1 & 0.0 & 19.1 & 36.5 & 24.2 & 20.2 & 88.2 & 10.9 & 0.8 & 0.1 & 99.8 & 0.2 & 0.0 & 0.0 \\
\hline & 400 & 93.1 & 6.3 & 0.5 & 0.1 & 95.1 & 4.5 & 0.4 & 0.1 & 0.0 & 21.0 & 36.9 & 23.8 & 18.4 & 93.7 & 6.0 & 0.3 & 0.0 & 100.0 & 0.0 & 0.0 & 0.0 \\
\hline \multirow[t]{4}{*}{0.1} & 50 & 77.6 & 19.0 & 2.9 & 0.6 & 96.9 & 2.7 & 0.4 & 0.0 & 0.0 & 6.8 & 24.5 & 28.9 & 39.8 & 41.3 & 37.9 & 14.4 & 6.5 & 88.4 & 10.6 & 0.9 & 0.1 \\
\hline & 100 & 88.4 & 10.3 & 1.1 & 0.2 & 96.1 & 3.5 & 0.4 & 0.0 & 0.0 & 13.8 & 32.7 & 27.0 & 26.5 & 73.3 & 22.3 & 3.6 & 0.8 & 98.7 & 1.4 & 0.0 & 0.0 \\
\hline & 200 & 91.2 & 7.9 & 0.8 & 0.2 & 95.5 & 4.1 & 0.3 & 0.1 & 0.0 & 18.7 & 35.3 & 25.3 & 20.7 & 88.0 & 10.9 & 1.0 & 0.1 & 99.8 & 0.2 & 0.0 & 0.0 \\
\hline & 400 & 93.3 & 6.2 & 0.5 & 0.1 & 95.4 & 4.2 & 0.4 & 0.1 & 0.0 & 21.4 & 36.8 & 24.1 & 17.8 & 93.7 & 6.0 & 0.3 & 0.1 & 100.0 & 0.0 & 0.0 & 0.0 \\
\hline \multirow[t]{4}{*}{0.2} & 50 & 74.4 & 21.6 & 3.3 & 0.7 & 96.7 & 2.9 & 0.4 & 0.1 & 0.0 & 5.5 & 23.2 & 28.4 & 42.9 & 37.1 & 38.8 & 16.1 & 8.0 & 86.4 & 12.6 & 1.0 & 0.1 \\
\hline & 100 & 87.1 & 11.3 & 1.4 & 0.3 & 95.9 & 3.7 & 0.4 & 0.0 & 0.0 & 12.8 & 32.9 & 26.6 & 27.7 & 71.3 & 23.9 & 4.0 & 0.8 & 98.3 & 1.7 & 0.0 & 0.0 \\
\hline & 200 & 91.1 & 8.0 & 0.8 & 0.1 & 95.3 & 4.2 & 0.4 & 0.1 & 0.0 & 17.3 & 35.4 & 25.4 & 21.9 & 87.0 & 12.0 & 1.0 & 0.1 & 99.8 & 0.2 & 0.0 & 0.0 \\
\hline & 400 & 93.1 & 6.1 & 0.6 & 0.2 & 95.6 & 4.1 & 0.2 & 0.0 & 0.0 & 20.5 & 36.0 & 24.4 & 19.1 & 93.7 & 5.9 & 0.3 & 0.0 & 100.0 & 0.1 & 0.0 & 0.0 \\
\hline \multirow[t]{4}{*}{0.3} & 50 & 70.6 & 23.7 & 4.7 & 1.1 & 96.8 & 2.9 & 0.2 & 0.1 & 0.0 & 4.3 & 19.9 & 27.6 & 48.2 & 34.0 & 37.8 & 18.4 & 9.8 & 84.8 & 13.6 & 1.4 & 0.2 \\
\hline & 100 & 85.5 & 12.6 & 1.8 & 0.3 & 95.7 & 3.9 & 0.4 & 0.1 & 0.0 & 11.9 & 30.6 & 27.9 & 29.6 & 70.7 & 24.1 & 4.2 & 1.0 & 98.2 & 1.7 & 0.0 & 0.0 \\
\hline & 200 & 90.6 & 8.2 & 1.1 & 0.2 & 95.8 & 3.8 & 0.4 & 0.0 & 0.0 & 17.2 & 35.1 & 25.1 & 22.6 & 86.4 & 12.3 & 1.2 & 0.2 & 99.8 & 0.2 & 0.0 & 0.0 \\
\hline & 400 & 92.7 & 6.5 & 0.7 & 0.1 & 95.5 & 4.1 & 0.3 & 0.1 & 0.0 & 20.9 & 35.9 & 24.3 & 18.9 & 93.2 & 6.6 & 0.2 & 0.0 & 100.0 & 0.0 & 0.0 & 0.0 \\
\hline \multirow[t]{4}{*}{0.5} & 50 & 59.2 & 30.7 & 8.0 & 2.1 & 96.4 & 3.3 & 0.3 & 0.1 & 0.0 & 2.1 & 13.3 & 24.7 & 59.9 & 24.3 & 37.5 & 21.1 & 17.1 & 78.9 & 18.3 & 2.2 & 0.6 \\
\hline & 100 & 80.8 & 16.2 & 2.5 & 0.5 & 95.6 & 3.9 & 0.4 & 0.1 & 0.0 & 8.7 & 26.9 & 28.6 & 35.8 & 65.2 & 27.1 & 5.8 & 1.9 & 97.7 & 2.2 & 0.1 & 0.0 \\
\hline & 200 & 88.2 & 10.5 & 1.1 & 0.2 & 95.7 & 3.9 & 0.3 & 0.1 & 0.0 & 15.0 & 33.2 & 26.2 & 25.6 & 85.3 & 13.2 & 1.4 & 0.2 & 99.7 & 0.3 & 0.0 & 0.0 \\
\hline & 400 & 91.6 & 7.4 & 0.8 & 0.2 & 95.2 & 4.4 & 0.4 & 0.0 & 0.0 & 19.6 & 35.7 & 24.2 & 20.5 & 93.3 & 6.4 & 0.3 & 0.0 & 100.0 & 0.1 & 0.0 & 0.0 \\
\hline \multirow[t]{4}{*}{0.8} & 50 & 18.2 & 36.9 & 27.0 & 17.9 & 94.8 & 4.5 & 0.6 & 0.1 & 0.3 & 0.1 & 1.5 & 7.4 & 91.0 & 6.4 & 17.4 & 20.8 & 55.4 & 58.7 & 27.7 & 8.0 & 5.6 \\
\hline & 100 & 52.9 & 33.6 & 10.1 & 3.4 & 95.0 & 4.4 & 0.5 & 0.1 & 0.1 & 1.9 & 11.7 & 22.4 & 64.1 & 43.3 & 35.4 & 13.9 & 7.4 & 93.9 & 5.8 & 0.2 & 0.1 \\
\hline & 200 & 76.4 & 19.6 & 3.2 & 0.9 & 94.9 & 4.6 & 0.4 & 0.1 & 0.0 & 7.1 & 25.0 & 27.3 & 40.7 & 75.2 & 21.0 & 3.2 & 0.6 & 99.5 & 0.5 & 0.0 & 0.0 \\
\hline & 400 & 87.3 & 11.0 & 1.4 & 0.3 & 95.2 & 4.3 & 0.5 & 0.0 & 0.0 & 14.1 & 32.3 & 26.5 & 27.1 & 90.1 & 9.2 & 0.6 & 0.0 & 100.0 & 0.0 & 0.0 & 0.0 \\
\hline \multirow[t]{4}{*}{0.9} & 50 & 5.8 & 22.1 & 30.5 & 41.5 & 93.8 & 5.3 & 0.8 & 0.1 & 2.2 & 0.0 & 0.3 & 1.5 & 98.2 & 2.3 & 7.6 & 11.0 & 79.1 & 46.1 & 27.8 & 11.2 & 14.9 \\
\hline & 100 & 23.8 & 37.7 & 23.7 & 14.8 & 93.8 & 5.4 & 0.8 & 0.1 & 0.2 & 0.2 & 2.5 & 8.8 & 88.5 & 23.2 & 30.4 & 19.4 & 27.0 & 88.8 & 9.7 & 1.1 & 0.4 \\
\hline & 200 & 55.1 & 31.5 & 10.2 & 3.3 & 94.7 & 4.7 & 0.5 & 0.1 & 0.0 & 2.3 & 12.6 & 23.1 & 62.1 & 61.3 & 29.0 & 6.8 & 2.9 & 98.9 & 1.1 & 0.0 & 0.0 \\
\hline & 400 & 77.3 & 19.0 & 3.0 & 0.7 & 94.6 & 4.7 & 0.6 & 0.1 & 0.1 & 7.2 & 25.5 & 28.3 & 39.1 & 84.5 & 13.7 & 1.6 & 0.3 & 99.9 & 0.1 & 0.0 & 0.0 \\
\hline
\end{tabular}


TABLE 1.3: Sequential procedures for determining the co-integration rank. VAR(2) model with rank $r_{0}=0$, GARCH $(1,1)$ errors [Case C]

\begin{tabular}{|c|c|c|c|c|c|c|c|c|c|c|c|c|c|c|c|c|c|c|c|c|c|c|}
\hline \multirow[b]{2}{*}{$\gamma$} & \multirow[b]{2}{*}{$T$} & \multicolumn{4}{|c|}{ Johansen } & \multicolumn{5}{|c|}{ Bootstrap } & \multicolumn{4}{|c|}{ AIC } & \multicolumn{4}{|c|}{ HQC } & \multicolumn{4}{|c|}{$\mathrm{BIC}$} \\
\hline & & $r=0$ & 1 & 2 & 3,4 & $r=0$ & 1 & 2 & 3,4 & $\mathrm{RC}$ & $r=0$ & 1 & 2 & 3,4 & $r=0$ & 1 & 2 & 3,4 & $r=0$ & 1 & 2 & 3,4 \\
\hline \multirow[t]{4}{*}{0.0} & 50 & 79.2 & 17.6 & 2.6 & 0.6 & 96.3 & 3.3 & 0.4 & 0.0 & 0.0 & 7.1 & 26.0 & 29.2 & 37.8 & 41.9 & 37.6 & 14.0 & 6.6 & 89.0 & 10.2 & 0.7 & 0.1 \\
\hline & 100 & 88.7 & 9.9 & 1.1 & 0.2 & 96.0 & 3.7 & 0.2 & 0.1 & 0.0 & 13.8 & 33.2 & 26.8 & 26.3 & 73.4 & 22.1 & 3.7 & 0.8 & 99.0 & 1.0 & 0.0 & 0.0 \\
\hline & 200 & 91.0 & 8.1 & 0.8 & 0.2 & 95.2 & 4.3 & 0.4 & 0.1 & 0.0 & 17.9 & 35.6 & 25.1 & 21.4 & 86.5 & 12.3 & 1.1 & 0.1 & 99.8 & 0.2 & 0.0 & 0.0 \\
\hline & 400 & 91.8 & 7.6 & 0.6 & 0.1 & 95.3 & 4.3 & 0.4 & 0.1 & 0.0 & 20.0 & 36.2 & 24.9 & 18.9 & 92.7 & 6.9 & 0.4 & 0.0 & 100.0 & 0.0 & 0.0 & 0.0 \\
\hline \multirow[t]{4}{*}{0.1} & 50 & 76.7 & 19.6 & 3.0 & 0.7 & 96.5 & 3.1 & 0.3 & 0.1 & 0.0 & 6.2 & 24.3 & 29.3 & 40.3 & 39.4 & 38.1 & 15.1 & 7.4 & 87.9 & 11.2 & 0.7 & 0.2 \\
\hline & 100 & 88.0 & 10.4 & 1.3 & 0.3 & 95.7 & 3.8 & 0.4 & 0.1 & 0.0 & 13.0 & 32.4 & 27.1 & 27.5 & 72.4 & 23.0 & 3.9 & 0.8 & 99.0 & 1.0 & 0.0 & 0.0 \\
\hline & 200 & 90.7 & 8.4 & 0.8 & 0.2 & 95.1 & 4.4 & 0.4 & 0.1 & 0.0 & 17.5 & 35.4 & 25.2 & 21.9 & 86.3 & 12.4 & 1.1 & 0.1 & 99.8 & 0.2 & 0.0 & 0.0 \\
\hline & 400 & 91.8 & 7.5 & 0.6 & 0.1 & 95.0 & 4.6 & 0.4 & 0.0 & 0.0 & 19.8 & 36.6 & 24.6 & 19.0 & 92.6 & 7.0 & 0.4 & 0.0 & 100.0 & 0.0 & 0.0 & 0.0 \\
\hline \multirow[t]{4}{*}{0.2} & 50 & 73.8 & 21.5 & 4.0 & 0.8 & 96.3 & 3.3 & 0.3 & 0.1 & 0.0 & 5.1 & 22.3 & 29.0 & 43.6 & 36.7 & 38.2 & 16.4 & 8.8 & 86.4 & 12.4 & 1.0 & 0.2 \\
\hline & 100 & 87.2 & 11.2 & 1.4 & 0.3 & 95.8 & 3.7 & 0.4 & 0.1 & 0.0 & 12.0 & 31.9 & 27.5 & 28.7 & 71.0 & 23.9 & 4.2 & 1.0 & 98.9 & 1.1 & 0.0 & 0.0 \\
\hline & 200 & 90.2 & 8.8 & 0.8 & 0.2 & 95.3 & 4.3 & 0.4 & 0.0 & 0.0 & 16.8 & 35.2 & 25.6 & 22.4 & 85.8 & 12.9 & 1.2 & 0.1 & 99.8 & 0.2 & 0.0 & 0.0 \\
\hline & 400 & 91.6 & 7.7 & 0.6 & 0.1 & 95.1 & 4.5 & 0.4 & 0.0 & 0.0 & 19.4 & 36.7 & 24.7 & 19.2 & 92.4 & 7.2 & 0.4 & 0.0 & 100.0 & 0.0 & 0.0 & 0.0 \\
\hline \multirow[t]{4}{*}{0.3} & 50 & 69.9 & 24.3 & 4.8 & 1.1 & 96.0 & 3.6 & 0.3 & 0.1 & 0.0 & 4.2 & 19.8 & 28.4 & 47.7 & 33.5 & 37.9 & 18.2 & 10.4 & 84.3 & 14.2 & 1.2 & 0.3 \\
\hline & 100 & 85.6 & 12.6 & 1.6 & 0.3 & 95.5 & 4.1 & 0.4 & 0.1 & 0.0 & 11.0 & 30.9 & 27.7 & 30.4 & 69.2 & 25.0 & 4.7 & 1.1 & 98.7 & 1.3 & 0.0 & 0.0 \\
\hline & 200 & 89.7 & 9.1 & 1.0 & 0.2 & 94.8 & 4.6 & 0.6 & 0.0 & 0.0 & 16.2 & 35.0 & 25.6 & 23.3 & 85.3 & 13.4 & 1.2 & 0.2 & 99.8 & 0.2 & 0.0 & 0.0 \\
\hline & 400 & 91.4 & 7.9 & 0.7 & 0.1 & 95.0 & 4.5 & 0.5 & 0.0 & 0.0 & 19.1 & 36.5 & 24.8 & 19.6 & 92.2 & 7.3 & 0.4 & 0.0 & 100.0 & 0.0 & 0.0 & 0.0 \\
\hline \multirow[t]{4}{*}{0.5} & 50 & 56.7 & 32.2 & 8.6 & 2.5 & 95.7 & 3.9 & 0.4 & 0.0 & 0.0 & 2.0 & 13.5 & 24.8 & 59.6 & 24.7 & 36.7 & 21.7 & 16.9 & 78.8 & 18.3 & 2.5 & 0.5 \\
\hline & 100 & 80.4 & 16.7 & 2.4 & 0.5 & 95.8 & 3.8 & 0.4 & 0.1 & 0.0 & 8.3 & 26.9 & 28.7 & 36.1 & 64.3 & 28.0 & 6.1 & 1.6 & 98.0 & 2.0 & 0.0 & 0.0 \\
\hline & 200 & 87.9 & 10.6 & 1.2 & 0.3 & 95.2 & 4.3 & 0.5 & 0.1 & 0.0 & 14.2 & 33.0 & 26.8 & 26.0 & 83.9 & 14.6 & 1.4 & 0.2 & 99.8 & 0.2 & 0.0 & 0.0 \\
\hline & 400 & 90.8 & 8.3 & 0.8 & 0.2 & 95.0 & 4.5 & 0.4 & 0.1 & 0.0 & 17.9 & 36.1 & 25.4 & 20.6 & 91.9 & 7.6 & 0.5 & 0.0 & 100.0 & 0.0 & 0.0 & 0.0 \\
\hline \multirow[t]{4}{*}{0.8} & 50 & 17.2 & 36.6 & 27.1 & 19.1 & 94.5 & 4.7 & 0.6 & 0.2 & 0.2 & 0.1 & 1.6 & 6.9 & 91.4 & 5.9 & 17.8 & 21.2 & 55.1 & 58.2 & 27.8 & 8.3 & 5.6 \\
\hline & 100 & 52.3 & 34.0 & 10.7 & 3.0 & 94.9 & 4.4 & 0.6 & 0.1 & 0.0 & 1.9 & 11.2 & 22.7 & 64.2 & 42.4 & 35.4 & 14.1 & 8.1 & 94.5 & 5.1 & 0.3 & 0.0 \\
\hline & 200 & 74.9 & 20.6 & 3.6 & 0.9 & 94.8 & 4.6 & 0.5 & 0.1 & 0.0 & 6.7 & 24.6 & 28.7 & 40.1 & 74.2 & 21.5 & 3.5 & 0.8 & 99.4 & 0.6 & 0.0 & 0.0 \\
\hline & 400 & 85.1 & 13.0 & 1.6 & 0.3 & 95.0 & 4.4 & 0.5 & 0.1 & 0.0 & 12.8 & 31.9 & 27.2 & 28.1 & 88.5 & 10.7 & 0.8 & 0.1 & 100.0 & 0.1 & 0.0 & 0.0 \\
\hline \multirow[t]{4}{*}{0.9} & 50 & 5.5 & 22.4 & 29.8 & 42.3 & 93.0 & 5.9 & 0.9 & 0.2 & 2.0 & 0.0 & 0.2 & 1.7 & 98.1 & 1.9 & 7.2 & 11.7 & 79.2 & 43.9 & 28.6 & 11.1 & 16.4 \\
\hline & 100 & 23.1 & 38.4 & 23.9 & 14.6 & 93.0 & 5.9 & 0.9 & 0.2 & 0.2 & 0.2 & 2.8 & 9.3 & 87.7 & 23.2 & 31.0 & 19.1 & 26.7 & 89.1 & 9.6 & 1.0 & 0.3 \\
\hline & 200 & 52.9 & 32.9 & 10.6 & 3.7 & 94.2 & 5.2 & 0.6 & 0.1 & 0.1 & 2.0 & 12.2 & 23.5 & 62.2 & 60.0 & 28.8 & 7.7 & 3.5 & 98.6 & 1.4 & 0.0 & 0.0 \\
\hline & 400 & 75.2 & 20.6 & 3.3 & 0.9 & 94.3 & 5.2 & 0.5 & 0.1 & 0.0 & 7.0 & 25.0 & 27.7 & 40.3 & 82.8 & 15.4 & 1.5 & 0.3 & 99.9 & 0.1 & 0.0 & 0.0 \\
\hline
\end{tabular}


TABLE 1.4: Sequential procedures for determining the co-integration rank. VAR(2) model with rank $r_{0}=0$, Autoregressive Stochastic Volatility [Case D]

\begin{tabular}{|c|c|c|c|c|c|c|c|c|c|c|c|c|c|c|c|c|c|c|c|c|c|c|}
\hline \multirow[b]{2}{*}{$\gamma$} & \multirow[b]{2}{*}{$T$} & \multicolumn{4}{|c|}{ Johansen } & \multicolumn{5}{|c|}{ Bootstrap } & \multicolumn{4}{|c|}{ AIC } & \multicolumn{4}{|c|}{ HQC } & \multicolumn{4}{|c|}{$\mathrm{BIC}$} \\
\hline & & $r=0$ & 1 & 2 & 3,4 & $r=0$ & 1 & 2 & 3,4 & $\mathrm{RC}$ & $r=0$ & 1 & 2 & 3,4 & $r=0$ & 1 & 2 & 3,4 & $r=0$ & 1 & 2 & 3,4 \\
\hline \multirow[t]{4}{*}{0.0} & 50 & 65.2 & 27.0 & 6.4 & 1.4 & 95.2 & 4.1 & 0.7 & 0.0 & 0.4 & 5.5 & 22.4 & 30.3 & 41.8 & 31.5 & 38.1 & 19.8 & 10.6 & 74.8 & 20.9 & 3.5 & 0.8 \\
\hline & 100 & 71.6 & 23.4 & 4.1 & 0.9 & 94.3 & 5.0 & 0.6 & 0.1 & 0.2 & 9.8 & 29.7 & 29.8 & 30.8 & 53.5 & 33.7 & 9.6 & 3.2 & 89.5 & 9.9 & 0.5 & 0.1 \\
\hline & 200 & 72.5 & 23.0 & 3.8 & 0.6 & 94.2 & 5.2 & 0.6 & 0.1 & 0.1 & 11.9 & 31.6 & 29.8 & 26.7 & 65.6 & 27.3 & 5.8 & 1.2 & 94.1 & 5.6 & 0.3 & 0.0 \\
\hline & 400 & 74.9 & 21.4 & 3.3 & 0.4 & 93.8 & 5.5 & 0.6 & 0.1 & 0.1 & 12.8 & 33.2 & 29.8 & 24.2 & 74.7 & 21.6 & 3.1 & 0.6 & 96.9 & 3.1 & 0.0 & 0.0 \\
\hline \multirow[t]{4}{*}{0.1} & 50 & 62.9 & 28.6 & 6.7 & 1.8 & 95.0 & 4.2 & 0.7 & 0.1 & 0.5 & 5.0 & 21.3 & 29.9 & 43.8 & 29.6 & 37.9 & 20.8 & 11.7 & 73.8 & 21.4 & 3.8 & 0.9 \\
\hline & 100 & 70.8 & 23.8 & 4.5 & 0.9 & 94.0 & 5.2 & 0.7 & 0.1 & 0.2 & 9.6 & 28.9 & 30.0 & 31.5 & 52.9 & 33.7 & 10.0 & 3.4 & 89.1 & 10.2 & 0.6 & 0.1 \\
\hline & 200 & 72.6 & 22.9 & 3.9 & 0.6 & 94.2 & 5.1 & 0.8 & 0.0 & 0.1 & 11.8 & 31.6 & 29.9 & 26.8 & 65.4 & 27.5 & 5.8 & 1.3 & 94.1 & 5.7 & 0.2 & 0.0 \\
\hline & 400 & 74.9 & 21.4 & 3.3 & 0.4 & 94.3 & 5.1 & 0.6 & 0.1 & 0.1 & 12.8 & 33.2 & 29.8 & 24.2 & 74.9 & 21.5 & 3.1 & 0.5 & 97.0 & 3.0 & 0.0 & 0.0 \\
\hline \multirow[t]{4}{*}{0.2} & 50 & 60.1 & 30.2 & 7.6 & 2.1 & 95.0 & 4.2 & 0.6 & 0.2 & 0.4 & 4.5 & 19.6 & 29.8 & 46.2 & 28.0 & 37.3 & 21.7 & 13.0 & 72.3 & 22.5 & 4.2 & 1.1 \\
\hline & 100 & 69.9 & 24.5 & 4.7 & 1.0 & 94.2 & 5.1 & 0.5 & 0.2 & 0.2 & 8.9 & 28.3 & 30.2 & 32.6 & 51.9 & 34.2 & 10.4 & 3.4 & 88.8 & 10.5 & 0.6 & 0.1 \\
\hline & 200 & 72.4 & 22.9 & 4.1 & 0.7 & 94.1 & 5.2 & 0.6 & 0.1 & 0.1 & 11.5 & 31.4 & 29.9 & 27.2 & 65.5 & 27.5 & 5.8 & 1.2 & 94.1 & 5.6 & 0.3 & 0.0 \\
\hline & 400 & 74.8 & 21.5 & 3.3 & 0.4 & 94.5 & 4.8 & 0.7 & 0.1 & 0.1 & 12.6 & 33.5 & 30.0 & 23.9 & 74.9 & 21.5 & 3.1 & 0.5 & 97.1 & 2.9 & 0.0 & 0.0 \\
\hline \multirow[t]{4}{*}{0.3} & 50 & 57.2 & 31.9 & 8.3 & 2.6 & 94.9 & 4.4 & 0.5 & 0.1 & 0.5 & 3.7 & 17.4 & 29.5 & 49.5 & 25.7 & 36.5 & 22.9 & 14.9 & 70.8 & 23.3 & 4.7 & 1.3 \\
\hline & 100 & 68.6 & 25.2 & 5.1 & 1.1 & 94.7 & 4.7 & 0.5 & 0.1 & 0.2 & 8.1 & 27.6 & 30.1 & 34.2 & 51.2 & 34.1 & 11.0 & 3.7 & 88.4 & 10.8 & 0.7 & 0.2 \\
\hline & 200 & 72.4 & 22.8 & 4.2 & 0.7 & 94.7 & 4.7 & 0.5 & 0.1 & 0.2 & 11.3 & 31.3 & 29.8 & 27.6 & 65.6 & 27.5 & 5.8 & 1.2 & 94.1 & 5.6 & 0.3 & 0.0 \\
\hline & 400 & 75.1 & 21.3 & 3.2 & 0.4 & 94.0 & 5.4 & 0.6 & 0.1 & 0.1 & 12.6 & 33.3 & 29.9 & 24.2 & 75.0 & 21.5 & 3.1 & 0.5 & 97.1 & 2.9 & 0.0 & 0.0 \\
\hline \multirow[t]{4}{*}{0.5} & 50 & 46.7 & 37.1 & 12.2 & 4.1 & 94.8 & 4.4 & 0.7 & 0.1 & 0.8 & 2.0 & 12.2 & 25.7 & 60.1 & 19.3 & 34.0 & 25.9 & 20.8 & 65.3 & 26.7 & 6.1 & 1.9 \\
\hline & 100 & 64.2 & 28.0 & 6.4 & 1.4 & 94.3 & 4.8 & 0.7 & 0.1 & 0.3 & 6.5 & 24.8 & 30.0 & 38.7 & 47.6 & 35.4 & 12.5 & 4.5 & 87.3 & 11.5 & 1.0 & 0.2 \\
\hline & 200 & 71.2 & 23.6 & 4.4 & 0.8 & 94.5 & 4.8 & 0.6 & 0.1 & 0.1 & 10.4 & 30.5 & 29.9 & 29.3 & 64.9 & 27.9 & 6.0 & 1.2 & 93.8 & 5.8 & 0.3 & 0.0 \\
\hline & 400 & 75.0 & 21.2 & 3.4 & 0.4 & 94.0 & 5.5 & 0.5 & 0.0 & 0.0 & 12.2 & 33.4 & 29.9 & 24.5 & 75.2 & 21.2 & 3.1 & 0.4 & 97.0 & 3.0 & 0.0 & 0.0 \\
\hline \multirow[t]{4}{*}{0.8} & 50 & 16.5 & 35.6 & 28.7 & 19.2 & 93.0 & 5.8 & 1.1 & 0.1 & 2.7 & 0.1 & 1.9 & 8.6 & 89.3 & 6.2 & 16.8 & 22.5 & 54.6 & 46.7 & 31.5 & 12.8 & 9.0 \\
\hline & 100 & 42.3 & 38.1 & 14.8 & 4.8 & 94.4 & 4.9 & 0.7 & 0.1 & 1.0 & 1.9 & 11.7 & 24.2 & 62.2 & 31.9 & 37.0 & 19.4 & 11.7 & 80.9 & 16.6 & 2.1 & 0.5 \\
\hline & 200 & 61.0 & 30.1 & 7.3 & 1.6 & 94.3 & 5.0 & 0.6 & 0.1 & 0.5 & 6.0 & 23.5 & 30.4 & 40.2 & 57.4 & 31.7 & 8.6 & 2.3 & 92.8 & 6.8 & 0.3 & 0.1 \\
\hline & 400 & 72.6 & 22.9 & 4.0 & 0.6 & 94.9 & 4.7 & 0.4 & 0.1 & 0.1 & 9.9 & 31.0 & 30.5 & 28.6 & 73.9 & 22.1 & 3.5 & 0.5 & 96.9 & 3.0 & 0.1 & 0.0 \\
\hline \multirow[t]{4}{*}{0.9} & 50 & 6.5 & 22.6 & 30.3 & 40.6 & 91.7 & 6.9 & 1.2 & 0.3 & 7.6 & 0.0 & 0.4 & 2.5 & 97.1 & 2.5 & 7.8 & 12.9 & 76.8 & 35.4 & 29.7 & 14.4 & 20.5 \\
\hline & 100 & 20.6 & 37.9 & 26.1 & 15.5 & 92.3 & 6.4 & 1.1 & 0.2 & 3.0 & 0.4 & 3.8 & 12.0 & 83.9 & 18.3 & 31.1 & 22.1 & 28.5 & 73.7 & 20.8 & 4.0 & 1.5 \\
\hline & 200 & 43.5 & 38.4 & 13.9 & 4.3 & 93.9 & 5.4 & 0.5 & 0.1 & 0.8 & 2.4 & 13.1 & 24.8 & 59.7 & 45.7 & 35.8 & 13.3 & 5.3 & 90.3 & 9.0 & 0.7 & 0.1 \\
\hline & 400 & 63.6 & 28.9 & 6.5 & 1.1 & 94.4 & 5.0 & 0.6 & 0.1 & 0.3 & 6.1 & 25.1 & 30.5 & 38.3 & 68.5 & 25.5 & 5.0 & 1.0 & 96.4 & 3.5 & 0.1 & 0.0 \\
\hline
\end{tabular}


TABLE 1.5: Sequential procedures for determining the co-integration rank. $\operatorname{VAR}(2)$ model with rank $r_{0}=0$, Single Volatility Break [Case E]

\begin{tabular}{|c|c|c|c|c|c|c|c|c|c|c|c|c|c|c|c|c|c|c|c|c|c|c|}
\hline \multirow[b]{2}{*}{$\gamma$} & \multirow[b]{2}{*}{$T$} & \multicolumn{4}{|c|}{ Johansen } & \multicolumn{5}{|c|}{ Bootstrap } & \multicolumn{4}{|c|}{ AIC } & \multicolumn{4}{|c|}{$\mathrm{HQC}$} & \multicolumn{4}{|c|}{ BIC } \\
\hline & & $r=0$ & 1 & 2 & 3,4 & $r=0$ & 1 & 2 & 3,4 & $\mathrm{RC}$ & $r=0$ & 1 & 2 & 3,4 & $r=0$ & 1 & 2 & 3,4 & $r=0$ & 1 & 2 & 3,4 \\
\hline \multirow[t]{4}{*}{0.0} & 50 & 42.9 & 41.9 & 12.9 & 2.3 & 90.0 & 8.5 & 1.4 & 0.2 & 0.6 & 1.4 & 13.1 & 30.4 & 55.2 & 14.5 & 36.0 & 30.5 & 19.0 & 57.8 & 32.7 & 7.8 & 1.7 \\
\hline & 100 & 53.2 & 36.5 & 8.8 & 1.5 & 92.0 & 7.1 & 0.8 & 0.1 & 0.3 & 2.6 & 19.0 & 32.9 & 45.5 & 33.8 & 41.6 & 17.8 & 6.7 & 85.5 & 13.3 & 1.1 & 0.1 \\
\hline & 200 & 58.5 & 32.9 & 7.4 & 1.2 & 92.9 & 6.4 & 0.6 & 0.1 & 0.2 & 3.9 & 21.8 & 33.2 & 41.2 & 53.0 & 34.2 & 10.1 & 2.7 & 95.7 & 4.2 & 0.1 & 0.0 \\
\hline & 400 & 59.9 & 32.7 & 6.5 & 0.8 & 94.5 & 4.9 & 0.5 & 0.1 & 0.1 & 4.3 & 22.6 & 34.7 & 38.4 & 64.1 & 29.4 & 5.4 & 1.1 & 98.6 & 1.4 & 0.0 & 0.0 \\
\hline \multirow[t]{4}{*}{0.1} & 50 & 41.4 & 42.3 & 13.7 & 2.6 & 90.3 & 8.4 & 1.2 & 0.1 & 0.7 & 1.3 & 12.1 & 30.0 & 56.6 & 13.6 & 35.1 & 31.0 & 20.4 & 56.3 & 33.4 & 8.4 & 1.9 \\
\hline & 100 & 51.8 & 37.5 & 9.2 & 1.6 & 91.9 & 7.0 & 0.9 & 0.1 & 0.4 & 2.5 & 18.2 & 33.0 & 46.4 & 32.9 & 41.9 & 18.4 & 6.8 & 85.1 & 13.7 & 1.1 & 0.2 \\
\hline & 200 & 58.1 & 32.9 & 7.8 & 1.3 & 93.2 & 6.0 & 0.8 & 0.1 & 0.3 & 4.0 & 21.5 & 32.9 & 41.6 & 52.3 & 34.4 & 10.3 & 2.9 & 95.7 & 4.1 & 0.2 & 0.0 \\
\hline & 400 & 59.5 & 33.2 & 6.5 & 0.8 & 93.9 & 5.5 & 0.6 & 0.1 & 0.2 & 4.2 & 22.7 & 34.3 & 38.8 & 63.8 & 29.5 & 5.6 & 1.1 & 98.5 & 1.5 & 0.0 & 0.0 \\
\hline \multirow[t]{4}{*}{0.2} & 50 & 39.3 & 43.2 & 14.8 & 2.8 & 89.4 & 9.1 & 1.3 & 0.3 & 0.6 & 1.1 & 11.4 & 28.9 & 58.6 & 12.7 & 34.0 & 31.6 & 21.8 & 54.9 & 34.1 & 9.0 & 2.1 \\
\hline & 100 & 50.4 & 38.4 & 9.5 & 1.7 & 91.9 & 7.2 & 0.8 & 0.2 & 0.5 & 2.4 & 17.7 & 33.0 & 47.0 & 31.8 & 42.3 & 18.7 & 7.2 & 84.4 & 14.2 & 1.2 & 0.2 \\
\hline & 200 & 57.5 & 33.5 & 7.7 & 1.3 & 93.2 & 6.0 & 0.7 & 0.1 & 0.2 & 3.9 & 21.1 & 32.9 & 42.2 & 51.4 & 34.9 & 10.6 & 3.1 & 95.5 & 4.2 & 0.2 & 0.0 \\
\hline & 400 & 59.3 & 33.3 & 6.6 & 0.8 & 94.0 & 5.4 & 0.5 & 0.1 & 0.2 & 4.1 & 22.3 & 34.4 & 39.2 & 63.7 & 29.6 & 5.7 & 1.0 & 98.5 & 1.5 & 0.0 & 0.0 \\
\hline \multirow[t]{4}{*}{0.3} & 50 & 36.6 & 44.1 & 16.0 & 3.3 & 90.6 & 8.2 & 1.1 & 0.2 & 0.8 & 0.9 & 10.5 & 28.2 & 60.4 & 11.4 & 33.2 & 32.2 & 23.3 & 53.0 & 34.8 & 9.9 & 2.2 \\
\hline & 100 & 48.7 & 39.3 & 10.2 & 1.8 & 91.7 & 7.2 & 0.9 & 0.2 & 0.5 & 2.2 & 16.9 & 32.7 & 48.2 & 30.7 & 42.3 & 19.4 & 7.6 & 83.6 & 14.9 & 1.3 & 0.2 \\
\hline & 200 & 56.4 & 34.2 & 8.1 & 1.3 & 93.3 & 5.9 & 0.8 & 0.0 & $0.3^{3}$ & 3.7 & 20.9 & 32.6 & 42.8 & 50.5 & 35.3 & 11.0 & 3.2 & 95.4 & 4.4 & 0.2 & 0.0 \\
\hline & 400 & 58.8 & 33.4 & 6.8 & 0.9 & 93.9 & 5.3 & 0.7 & 0.1 & 0.3 & 4.0 & 22.1 & 34.5 & 39.5 & 63.1 & 30.0 & 5.8 & 1.1 & 98.5 & 1.5 & 0.0 & 0.0 \\
\hline \multirow[t]{4}{*}{0.5} & 50 & 29.8 & 45.9 & 19.8 & 4.6 & 89.8 & 8.9 & 1.3 & 0.1 & 1.0 & 0.5 & 7.6 & 25.1 & 66.8 & 8.9 & 29.5 & 33.6 & 28.0 & 48.3 & 36.5 & 12.0 & 3.3 \\
\hline & 100 & 44.6 & 41.1 & 12.1 & 2.2 & 91.2 & 7.6 & 1.1 & 0.1 & 0.4 & 1.8 & 14.6 & 32.1 & 51.5 & 27.9 & 42.1 & 20.9 & 9.1 & 81.8 & 16.2 & 1.8 & 0.2 \\
\hline & 200 & 53.5 & 36.0 & 9.0 & 1.5 & 93.3 & 6.0 & 0.7 & 0.1 & 0.3 & 3.1 & 19.8 & 32.9 & 44.2 & 48.0 & 36.9 & 11.6 & 3.4 & 94.9 & 4.9 & 0.3 & 0.0 \\
\hline & 400 & 58.0 & 33.6 & 7.5 & 0.9 & 94.4 & 5.1 & 0.5 & 0.1 & 0.3 & 3.6 & 21.3 & 34.6 & 40.5 & 62.0 & 30.5 & 6.2 & 1.2 & 98.4 & 1.6 & 0.0 & 0.0 \\
\hline \multirow[t]{4}{*}{0.8} & 50 & 12.0 & 40.1 & 33.5 & 14.4 & 88.5 & 10.4 & 1.0 & 0.1 & 5.9 & 0.1 & 2.2 & 13.0 & 84.8 & 3.2 & 17.1 & 29.5 & 50.3 & 33.5 & 39.3 & 18.9 & 8.3 \\
\hline & 100 & 29.4 & 46.0 & 19.5 & 5.1 & 92.0 & 7.1 & 0.8 & 0.1 & 0.7 & 0.7 & 7.7 & 26.7 & 64.9 & 19.1 & 39.1 & 27.1 & 14.7 & 73.9 & 22.0 & 3.6 & 0.5 \\
\hline & 200 & 42.8 & 40.7 & 14.0 & 2.5 & 93.8 & 5.6 & 0.6 & 0.1 & 0.6 & 1.7 & 14.5 & 31.3 & 52.5 & 40.1 & 39.2 & 15.6 & 5.2 & 92.2 & 7.4 & 0.4 & 0.0 \\
\hline & 400 & 50.3 & 38.4 & 9.6 & 1.6 & 94.1 & 5.3 & 0.5 & 0.1 & 0.4 & 2.8 & 18.7 & 34.2 & 44.3 & 56.4 & 33.5 & 8.3 & 1.9 & 97.9 & 2.1 & 0.0 & 0.0 \\
\hline \multirow[t]{4}{*}{0.9} & 50 & 4.9 & 27.6 & 37.0 & 30.6 & 86.9 & 11.5 & 1.5 & 0.1 & 18.8 & 0.0 & 0.7 & 5.2 & 94.1 & 1.3 & 9.0 & 20.9 & 68.8 & 24.6 & 35.5 & 22.2 & 17.7 \\
\hline & 100 & 16.0 & 42.4 & 29.7 & 11.9 & 90.7 & 8.3 & 0.9 & 0.1 & 4.5 & 0.2 & 3.5 & 16.8 & 79.5 & 12.0 & 32.1 & 29.8 & 26.2 & 65.5 & 27.3 & 6.0 & 1.2 \\
\hline & 200 & 30.7 & 44.4 & 19.8 & 5.1 & 93.0 & 6.3 & 0.6 & 0.1 & 0.8 & 0.9 & 8.9 & 27.2 & 63.1 & 32.0 & 40.0 & 19.9 & 8.2 & 89.2 & 10.1 & 0.6 & 0.0 \\
\hline & 400 & 41.7 & 43.0 & 12.9 & 2.5 & 94.7 & 4.8 & 0.5 & 0.1 & 0.4 & 1.7 & 14.8 & 32.2 & 51.4 & 50.0 & 36.6 & 10.9 & 2.6 & 97.3 & 2.6 & 0.1 & 0.0 \\
\hline
\end{tabular}


TABLE 2.1: Sequential procedures for determining the co-integration rank. $\operatorname{VAR}(2)$ model with rank $r_{0}=1$, i.i.d. Gaussian errors [Case A]

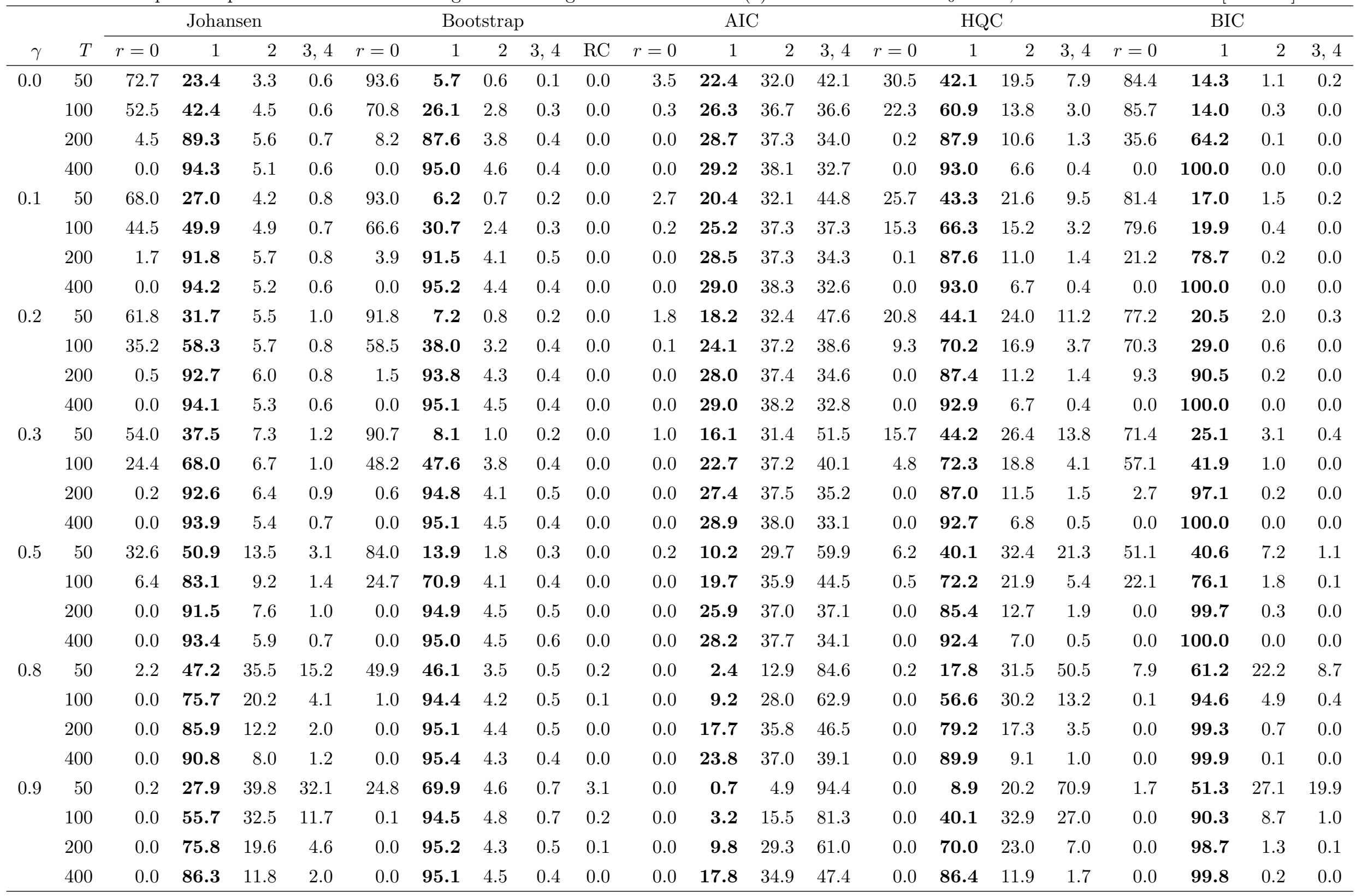


TABLE 2.2: Sequential procedures for determining the co-integration rank. VAR(2) model with rank $r_{0}=1$, i.i.d. $t(5)$ errors [Case B]

\begin{tabular}{|c|c|c|c|c|c|c|c|c|c|c|c|c|c|c|c|c|c|c|c|c|c|c|}
\hline \multirow[b]{2}{*}{$\gamma$} & \multirow[b]{2}{*}{$T$} & \multicolumn{4}{|c|}{ Johansen } & \multicolumn{5}{|c|}{ Bootstrap } & \multicolumn{4}{|c|}{ AIC } & \multicolumn{4}{|c|}{ HQC } & \multicolumn{4}{|c|}{$\mathrm{BIC}$} \\
\hline & & $r=0$ & 1 & 2 & 3,4 & $r=0$ & 1 & 2 & 3,4 & $\mathrm{RC}$ & $r=0$ & 1 & 2 & 3,4 & $r=0$ & 1 & 2 & 3,4 & $r=0$ & 1 & 2 & 3,4 \\
\hline \multirow[t]{3}{*}{0.0} & 50 & 72.1 & 23.7 & 3.5 & 0.6 & 94.6 & 4.8 & 0.6 & 0.1 & 0.0 & 3.4 & 22.4 & 32.8 & 41.4 & 30.7 & 41.5 & 19.0 & 8.8 & 83.4 & 15.1 & 1.4 & 0.2 \\
\hline & 100 & 51.2 & 43.3 & 4.9 & 0.6 & 71.1 & 26.2 & 2.3 & 0.4 & 0.0 & 0.3 & 25.6 & 37.0 & 37.1 & 20.3 & 62.1 & 14.3 & 3.3 & 84.8 & 14.9 & 0.3 & 0.0 \\
\hline & 400 & 0.0 & 94.6 & 4.9 & 0.5 & 0.0 & 95.1 & 4.4 & 0.5 & 0.0 & 0.0 & 29.8 & 38.3 & 32.0 & 0.0 & 92.8 & 6.8 & 0.5 & 0.0 & 100.0 & 0.0 & 0.0 \\
\hline \multirow[t]{3}{*}{0.1} & 50 & 66.9 & 27.7 & 4.5 & 0.9 & 93.4 & 5.8 & 0.6 & 0.2 & 0.0 & 2.2 & 20.7 & 32.7 & 44.4 & 25.5 & 43.4 & 21.1 & 10.0 & 80.0 & 18.0 & 1.7 & 0.3 \\
\hline & 100 & 43.0 & 50.7 & 5.5 & 0.8 & 65.5 & 31.6 & 2.6 & 0.4 & 0.0 & 0.1 & 24.9 & 37.3 & 37.7 & 13.6 & 66.8 & 15.9 & 3.7 & 78.9 & 20.7 & 0.4 & 0.0 \\
\hline & 400 & 0.0 & 94.0 & 5.5 & 0.6 & 0.0 & 95.0 & 4.6 & 0.4 & 0.0 & 0.0 & 30.7 & 38.3 & 31.0 & 0.0 & 92.8 & 6.7 & 0.6 & 0.0 & 100.0 & 0.1 & 0.0 \\
\hline \multirow[t]{4}{*}{0.2} & 50 & 61.0 & 31.9 & 5.7 & 1.3 & 91.8 & 7.4 & 0.7 & 0.2 & 0.0 & 1.7 & 17.8 & 33.3 & 47.2 & 21.2 & 43.6 & 24.0 & 11.3 & 76.3 & 21.2 & 2.2 & 0.3 \\
\hline & 100 & 33.5 & 59.1 & 6.4 & 1.0 & 58.5 & 38.0 & 3.0 & 0.5 & 0.0 & 0.0 & 24.0 & 37.5 & 38.5 & 8.3 & 69.4 & 18.7 & 3.7 & 69.4 & 30.0 & 0.6 & 0.0 \\
\hline & 200 & 0.4 & 92.1 & 6.8 & 0.8 & 2.2 & 93.3 & 4.0 & 0.6 & 0.0 & 0.0 & 28.1 & 37.8 & 34.1 & 0.0 & 86.4 & 12.2 & 1.4 & 8.5 & 91.2 & 0.3 & 0.0 \\
\hline & 400 & 0.0 & 93.6 & 5.8 & 0.5 & 0.0 & 95.6 & 4.0 & 0.4 & 0.0 & 0.0 & 30.3 & 37.6 & 32.1 & 0.0 & 92.4 & 7.2 & 0.5 & 0.0 & 100.0 & 0.0 & 0.0 \\
\hline 0.3 & 50 & 53.9 & 37.0 & 7.6 & 1.4 & 90.9 & 8.0 & 0.9 & 0.2 & 0.0 & 1.0 & 16.7 & 31.6 & 50.7 & 16.0 & 44.3 & 26.3 & 13.5 & 70.3 & 26.2 & 3.0 & 0.5 \\
\hline \multirow{3}{*}{0.5} & 100 & 6.5 & 82.3 & 9.9 & 1.3 & 25.2 & 70.6 & 3.8 & 0.4 & 0.0 & 0.0 & 19.4 & 35.8 & 44.8 & 0.4 & 71.7 & 22.4 & 5.5 & 20.9 & 76.9 & 2.1 & 0.1 \\
\hline & 200 & 0.0 & 91.6 & 7.5 & 0.9 & 0.1 & 95.6 & 3.9 & 0.4 & 0.0 & 0.0 & 25.2 & 37.0 & 37.8 & 0.0 & 85.1 & 13.0 & 1.9 & 0.0 & 99.5 & 0.5 & 0.0 \\
\hline & 400 & 0.0 & 93.0 & 6.3 & 0.7 & 0.0 & 95.1 & 4.5 & 0.4 & 0.0 & 0.0 & 28.8 & 38.2 & 33.1 & 0.0 & 92.2 & 7.3 & 0.5 & 0.0 & 100.0 & 0.1 & 0.0 \\
\hline \multirow[t]{4}{*}{0.8} & 50 & 1.8 & 48.4 & 34.9 & 15.0 & 49.8 & 46.1 & 3.7 & 0.4 & 0.4 & 0.0 & 2.0 & 12.9 & 85.2 & 0.2 & 18.2 & 30.1 & 51.5 & 6.8 & 61.7 & 22.6 & 9.0 \\
\hline & 100 & 0.0 & 74.9 & 21.0 & 4.0 & 1.2 & 94.3 & 4.0 & 0.5 & 0.1 & 0.0 & 9.4 & 27.7 & 62.9 & 0.0 & 55.9 & 30.2 & 14.0 & 0.1 & 93.9 & 5.5 & 0.5 \\
\hline & 200 & 0.0 & 85.8 & 12.3 & 1.9 & 0.0 & 95.0 & 4.5 & 0.5 & 0.0 & 0.0 & 17.9 & 35.0 & 47.2 & 0.0 & 79.3 & 17.3 & 3.5 & 0.0 & 99.2 & 0.8 & 0.0 \\
\hline & 400 & 0.0 & 91.0 & 7.9 & 1.1 & 0.0 & 95.1 & 4.5 & 0.5 & 0.1 & 0.0 & 24.6 & 37.0 & 38.4 & 0.0 & 89.9 & 9.2 & 0.9 & 0.0 & 99.8 & 0.2 & 0.0 \\
\hline \multirow[t]{4}{*}{0.9} & 50 & 0.1 & 28.5 & 40.3 & 31.1 & 23.4 & 71.5 & 4.6 & 0.6 & 3.3 & 0.0 & 0.6 & 4.9 & 94.5 & 0.0 & 9.3 & 20.3 & 70.4 & 1.7 & 52.4 & 26.4 & 19.5 \\
\hline & 100 & 0.0 & 55.3 & 32.8 & 11.9 & 0.1 & 94.8 & 4.6 & 0.5 & 0.3 & 0.0 & 3.2 & 15.5 & 81.2 & 0.0 & 40.2 & 32.5 & 27.3 & 0.0 & 89.6 & 9.2 & 1.3 \\
\hline & 200 & 0.0 & 75.7 & 20.0 & 4.3 & 0.0 & 94.9 & 4.6 & 0.5 & 0.1 & 0.0 & 9.8 & 28.0 & 62.2 & 0.0 & 69.6 & 23.4 & 7.0 & 0.0 & 98.4 & 1.5 & 0.0 \\
\hline & 400 & 0.0 & 86.9 & 11.3 & 1.8 & 0.0 & 94.7 & 4.8 & 0.5 & 0.1 & 0.0 & 17.8 & 35.9 & 46.3 & 0.0 & 86.3 & 12.0 & 1.7 & 0.0 & 99.9 & 0.1 & 0.0 \\
\hline
\end{tabular}


TABLE 2.3: Sequential procedures for determining the co-integration rank. VAR(2) model with rank $r_{0}=1$, GARCH(1,1) errors [Case C

\begin{tabular}{|c|c|c|c|c|c|c|c|c|c|c|c|c|c|c|c|c|c|c|c|c|c|c|}
\hline \multirow[b]{2}{*}{$\gamma$} & \multirow[b]{2}{*}{$T$} & \multicolumn{4}{|c|}{ Johansen } & \multicolumn{5}{|c|}{ Bootstrap } & \multicolumn{4}{|c|}{ AIC } & \multicolumn{4}{|c|}{ HQC } & \multicolumn{4}{|c|}{ BIC } \\
\hline & & $r=0$ & 1 & 2 & 3,4 & $r=0$ & 1 & 2 & 3,4 & $\mathrm{RC}$ & $r=0$ & 1 & 2 & 3,4 & $r=0$ & 1 & 2 & 3,4 & $r=0$ & 1 & 2 & 3,4 \\
\hline \multirow{2}{*}{0.0} & 100 & 52.3 & 42.0 & 4.9 & 0.8 & 70.9 & 26.3 & 2.5 & 0.4 & 0.0 & 0.4 & 25.6 & 37.1 & 37.0 & 21.7 & 60.3 & 14.9 & 3.2 & 86.4 & 13.3 & 0.3 & 0.0 \\
\hline & 400 & 0.0 & 92.7 & 6.6 & 0.7 & 0.0 & 94.7 & 4.9 & 0.4 & 0.0 & 0.0 & 29.3 & 38.3 & 32.4 & 0.0 & 90.9 & 8.3 & 0.7 & 0.1 & 99.8 & 0.1 & 0.0 \\
\hline \multirow[t]{3}{*}{0.1} & 50 & 68.2 & 26.8 & 4.3 & 0.8 & 93.1 & 6.1 & 0.7 & 0.1 & 0.0 & 2.3 & 19.9 & 33.1 & 44.7 & 27.0 & 42.3 & 21.3 & 9.4 & 81.4 & 17.0 & 1.5 & 0.2 \\
\hline & 100 & 44.0 & 49.6 & 5.6 & 0.9 & 65.2 & 31.5 & 2.8 & 0.5 & 0.0 & 0.2 & 24.8 & 37.0 & 38.0 & 15.2 & 64.8 & 16.5 & 3.5 & 80.1 & 19.4 & 0.5 & 0.0 \\
\hline & 400 & 0.0 & 92.7 & 6.6 & 0.7 & 0.0 & 94.8 & 4.7 & 0.6 & 0.1 & 0.0 & 29.2 & 38.1 & 32.8 & 0.0 & 90.9 & 8.4 & 0.7 & 0.0 & 99.9 & 0.1 & 0.0 \\
\hline \multirow[t]{4}{*}{0.2} & 50 & 62.2 & 31.1 & 5.6 & 1.1 & 91.8 & 7.3 & 0.8 & 0.2 & 0.0 & 1.6 & 18.0 & 32.8 & 47.6 & 22.3 & 42.3 & 24.0 & 11.4 & 77.3 & 20.5 & 2.1 & 0.2 \\
\hline & 100 & 34.9 & 57.8 & 6.4 & 1.0 & 57.8 & 38.5 & 3.3 & 0.4 & 0.0 & 0.1 & 23.8 & 37.1 & 39.1 & 9.0 & 69.1 & 17.9 & 4.0 & 71.1 & 28.3 & 0.6 & 0.0 \\
\hline & 200 & 0.5 & 91.8 & 6.9 & 0.8 & 2.2 & 93.4 & 3.8 & 0.5 & 0.0 & 0.0 & 27.5 & 37.3 & 35.3 & 0.0 & 85.9 & 12.6 & 1.5 & 9.9 & 89.7 & 0.4 & 0.0 \\
\hline & 400 & 0.0 & 92.6 & 6.7 & 0.8 & 0.0 & 95.3 & 4.3 & 0.4 & 0.0 & 0.0 & 28.9 & 38.1 & 33.0 & 0.0 & 90.8 & 8.5 & 0.7 & 0.0 & 99.9 & 0.1 & 0.0 \\
\hline 0.3 & 50 & 54.5 & 36.8 & 7.2 & 1.5 & 90.6 & 8.1 & 1.1 & 0.2 & 0.0 & 1.0 & 15.3 & 32.3 & 51.5 & 16.9 & 42.4 & 26.5 & 14.2 & 71.6 & 24.9 & 3.2 & 0.3 \\
\hline \multirow{3}{*}{0.5} & 100 & 6.5 & 82.0 & 10.2 & 1.4 & 26.1 & 69.6 & 3.9 & 0.4 & 0.0 & 0.0 & 19.1 & 36.2 & 44.7 & 0.5 & 71.2 & 22.3 & 6.0 & 22.2 & 75.7 & 2.0 & 0.1 \\
\hline & 200 & 0.0 & 91.2 & 7.8 & 1.0 & 0.0 & 95.0 & 4.5 & 0.5 & 0.0 & 0.0 & 24.8 & 37.5 & 37.7 & 0.0 & 84.3 & 13.9 & 1.8 & 0.1 & 99.4 & 0.5 & 0.0 \\
\hline & 400 & 0.0 & 91.9 & 7.3 & 0.8 & 0.0 & 95.5 & 4.1 & 0.4 & 0.1 & 0.0 & 27.7 & 38.2 & 34.1 & 0.0 & 90.4 & 8.7 & 0.9 & 0.0 & 99.9 & 0.1 & 0.0 \\
\hline \multirow[t]{4}{*}{0.8} & 50 & 2.2 & 47.0 & 35.5 & 15.3 & 49.8 & 46.2 & 3.7 & 0.5 & 0.3 & 0.0 & 2.1 & 12.7 & 85.2 & 0.2 & 18.2 & 31.0 & 50.6 & 7.9 & 60.9 & 22.5 & 8.8 \\
\hline & 100 & 0.0 & 74.7 & 20.9 & 4.3 & 1.1 & 94.3 & 4.3 & 0.4 & 0.1 & 0.0 & 8.8 & 27.3 & 63.8 & 0.0 & 55.6 & 30.7 & 13.7 & 0.1 & 94.3 & 5.2 & 0.4 \\
\hline & 200 & 0.0 & 85.5 & 12.6 & 1.9 & 0.0 & 94.7 & 4.9 & 0.4 & 0.0 & 0.0 & 16.8 & 35.2 & 48.0 & 0.0 & 78.0 & 18.3 & 3.7 & 0.0 & 99.0 & 1.0 & 0.0 \\
\hline & 400 & 0.0 & 89.5 & 9.2 & 1.3 & 0.0 & 95.4 & 4.2 & 0.5 & 0.0 & 0.0 & 23.4 & 37.8 & 38.8 & 0.0 & 87.9 & 10.7 & 1.4 & 0.0 & 99.9 & 0.2 & 0.0 \\
\hline \multirow[t]{4}{*}{0.9} & 50 & 0.2 & 27.6 & 39.8 & 32.3 & 24.8 & 69.6 & 5.0 & 0.6 & 3.3 & 0.0 & 0.5 & 5.0 & 94.6 & 0.0 & 8.8 & 19.9 & 71.3 & 1.8 & 50.3 & 27.6 & 20.3 \\
\hline & 100 & 0.0 & 55.6 & 32.4 & 12.1 & 0.1 & 94.5 & 4.9 & 0.6 & 0.3 & 0.0 & 3.2 & 16.2 & 80.6 & 0.0 & 39.2 & 33.4 & 27.5 & 0.0 & 89.6 & 9.0 & 1.4 \\
\hline & 200 & 0.0 & 74.6 & 20.9 & 4.6 & 0.0 & 94.5 & 4.9 & 0.6 & 0.1 & 0.0 & 9.5 & 28.2 & 62.3 & 0.0 & 68.1 & 24.3 & 7.6 & 0.0 & $\mathbf{9 8 . 3}$ & 1.7 & 0.1 \\
\hline & 400 & 0.0 & 85.2 & 12.9 & 1.9 & 0.0 & 94.5 & 5.0 & 0.4 & 0.0 & 0.0 & 17.8 & 35.6 & 46.7 & 0.0 & 84.3 & 13.6 & 2.0 & 0.0 & 99.7 & 0.3 & 0.0 \\
\hline
\end{tabular}


TABLE 2.4: Sequential procedures for determining the co-integration rank. $\operatorname{VAR}(2)$ model with rank $r_{0}=1$, Autoregressive Stochastic Volatility [Case D]

\begin{tabular}{|c|c|c|c|c|c|c|c|c|c|c|c|c|c|c|c|c|c|c|c|c|c|c|}
\hline \multirow[b]{2}{*}{$\gamma$} & \multirow[b]{2}{*}{$T$} & \multicolumn{4}{|c|}{ Johansen } & \multicolumn{5}{|c|}{ Bootstrap } & \multicolumn{4}{|c|}{ AIC } & \multicolumn{4}{|c|}{ HQC } & \multicolumn{4}{|c|}{$\mathrm{BIC}$} \\
\hline & & $r=0$ & 1 & 2 & 3,4 & $r=0$ & 1 & 2 & 3,4 & $\mathrm{RC}$ & $r=0$ & 1 & 2 & 3,4 & $r=0$ & 1 & 2 & 3,4 & $r=0$ & 1 & 2 & 3,4 \\
\hline \multirow{2}{*}{0.0} & 100 & 37.8 & 47.4 & 12.1 & 2.7 & 73.3 & 22.0 & 4.1 & 0.7 & 0.2 & 0.9 & 19.6 & 35.8 & 43.7 & 19.1 & 49.6 & 23.3 & 8.1 & 70.0 & 26.1 & 3.5 & 0.5 \\
\hline & 400 & 0.1 & 79.7 & 17.8 & 2.4 & 2.9 & 91.0 & 5.7 & 0.5 & 0.1 & 0.0 & 21.4 & 39.0 & 39.7 & 0.1 & 76.2 & 20.5 & 3.2 & 2.3 & 94.2 & 3.5 & 0.1 \\
\hline \multirow[t]{3}{*}{0.1} & 50 & 53.7 & 34.1 & 9.6 & 2.6 & 91.1 & 7.2 & 1.4 & 0.3 & 0.4 & 2.4 & 17.2 & 32.1 & 48.3 & 20.4 & 38.3 & 25.8 & 15.5 & 66.3 & 26.6 & 5.5 & 1.6 \\
\hline & 100 & 32.4 & 51.2 & 13.5 & 2.8 & 70.3 & 25.3 & 3.8 & 0.7 & 0.3 & 0.6 & 19.1 & 36.1 & 44.2 & 15.1 & 51.6 & 24.7 & 8.6 & 64.6 & 30.6 & 4.2 & 0.6 \\
\hline & 400 & 0.1 & 79.9 & 17.7 & 2.4 & 1.8 & 92.5 & 5.1 & 0.6 & 0.2 & 0.0 & 21.3 & 39.0 & 39.7 & 0.0 & 76.3 & 20.6 & 3.1 & 1.2 & 95.3 & 3.5 & 0.1 \\
\hline \multirow[t]{4}{*}{0.2} & 50 & 48.8 & 37.0 & 11.3 & 3.0 & 89.8 & 8.5 & 1.4 & 0.3 & 0.3 & 1.8 & 15.5 & 32.1 & 50.7 & 17.3 & 37.6 & 27.8 & 17.3 & 62.2 & 29.1 & 6.8 & 1.9 \\
\hline & 100 & 26.6 & 55.3 & 15.1 & 3.1 & 64.9 & 30.3 & 4.2 & 0.7 & 0.3 & 0.4 & 18.5 & 36.0 & 45.0 & 10.8 & 53.4 & 26.5 & 9.3 & 57.1 & 37.1 & 5.0 & 0.8 \\
\hline & 200 & 2.7 & 75.9 & 18.5 & 2.9 & 17.7 & 75.2 & 6.2 & 0.8 & 0.3 & 0.0 & 20.3 & 37.9 & 41.7 & 1.0 & 68.4 & 24.7 & 5.9 & 16.5 & 77.5 & 5.6 & 0.4 \\
\hline & 400 & 0.0 & 80.0 & 17.6 & 2.3 & 1.0 & 92.9 & 5.6 & 0.5 & 0.1 & 0.0 & 21.3 & 39.1 & 39.6 & 0.0 & 76.4 & 20.5 & 3.1 & 0.5 & 95.8 & 3.6 & 0.1 \\
\hline 0.3 & 50 & 42.8 & 40.2 & 13.3 & 3.7 & 88.2 & 10.0 & 1.6 & 0.3 & 0.5 & 1.2 & 13.6 & 31.6 & 53.6 & 13.9 & 36.6 & 30.1 & 19.3 & 57.2 & 32.2 & 8.3 & 2.3 \\
\hline \multirow{3}{*}{0.5} & 100 & 8.4 & 67.4 & 20.1 & 4.1 & 41.4 & 52.7 & 5.4 & 0.6 & 0.2 & 0.1 & 16.1 & 35.1 & 48.8 & 2.2 & 55.3 & 30.6 & 11.9 & 24.1 & 65.9 & 8.6 & 1.4 \\
\hline & 200 & 0.3 & 77.6 & 19.2 & 2.9 & 4.3 & 89.1 & 6.1 & 0.5 & 0.3 & 0.0 & 19.6 & 38.0 & 42.5 & 0.1 & 68.4 & 25.4 & 6.1 & 2.5 & 91.0 & 6.1 & 0.4 \\
\hline & 400 & 0.0 & 80.1 & 17.8 & 2.1 & 0.2 & 94.3 & 4.9 & 0.6 & 0.1 & 0.0 & 21.3 & 39.0 & 39.7 & 0.0 & 76.8 & 20.2 & 3.0 & 0.0 & 96.3 & 3.6 & 0.1 \\
\hline \multirow[t]{4}{*}{0.8} & 50 & 3.3 & 41.9 & 37.1 & 17.7 & 54.8 & 39.9 & 4.7 & 0.6 & 3.3 & 0.0 & 1.9 & 14.7 & 83.4 & 0.6 & 16.7 & 30.8 & 51.9 & 9.6 & 50.6 & 26.8 & 13.0 \\
\hline & 100 & 0.5 & 63.0 & 28.8 & 7.7 & 8.7 & 84.7 & 5.8 & 0.8 & 1.4 & 0.0 & 8.9 & 28.0 & 63.1 & 0.1 & 45.5 & 35.5 & 19.0 & 1.8 & 81.4 & 14.5 & 2.4 \\
\hline & 200 & 0.0 & 73.4 & 22.7 & 3.9 & 0.5 & 94.1 & 4.9 & 0.6 & 0.4 & 0.0 & 15.6 & 36.0 & 48.5 & 0.0 & 64.8 & 27.8 & 7.4 & 0.1 & 92.4 & 7.0 & 0.5 \\
\hline & 400 & 0.0 & 79.9 & 17.5 & 2.6 & 0.0 & 94.2 & 5.2 & 0.6 & 0.2 & 0.0 & 20.0 & 38.8 & 41.2 & 0.0 & 76.6 & 20.2 & 3.2 & 0.0 & 96.6 & 3.3 & 0.1 \\
\hline \multirow[t]{4}{*}{0.9} & 50 & 0.5 & 26.6 & 39.4 & 33.6 & 32.8 & 60.3 & 5.8 & 1.1 & 8.7 & 0.0 & 0.5 & 6.5 & 93.0 & 0.1 & 8.4 & 22.1 & 69.4 & 3.3 & 43.2 & 29.3 & 24.2 \\
\hline & 100 & 0.0 & 47.7 & 36.8 & 15.5 & 2.9 & 90.2 & 6.1 & 0.8 & 3.0 & 0.0 & 3.7 & 18.1 & 78.2 & 0.0 & 33.1 & 35.9 & 31.0 & 0.4 & 76.7 & 18.5 & 4.4 \\
\hline & 200 & 0.0 & 64.6 & 28.7 & 6.7 & 0.1 & 94.3 & 4.9 & 0.6 & 1.1 & 0.0 & 9.7 & 29.6 & 60.8 & 0.0 & 57.0 & 32.1 & 10.9 & 0.0 & 90.6 & 8.7 & 0.7 \\
\hline & 400 & 0.0 & 76.6 & 20.2 & 3.3 & 0.0 & 94.8 & 4.8 & 0.4 & 0.4 & 0.0 & 16.5 & 37.1 & 46.4 & 0.0 & 73.8 & 22.3 & 4.0 & 0.0 & 96.1 & 3.7 & 0.1 \\
\hline
\end{tabular}


TABLE 2.5: Sequential procedures for determining the co-integration rank. VAR(2) model with rank $r_{0}=1$, Single Volatility Break [Case E]

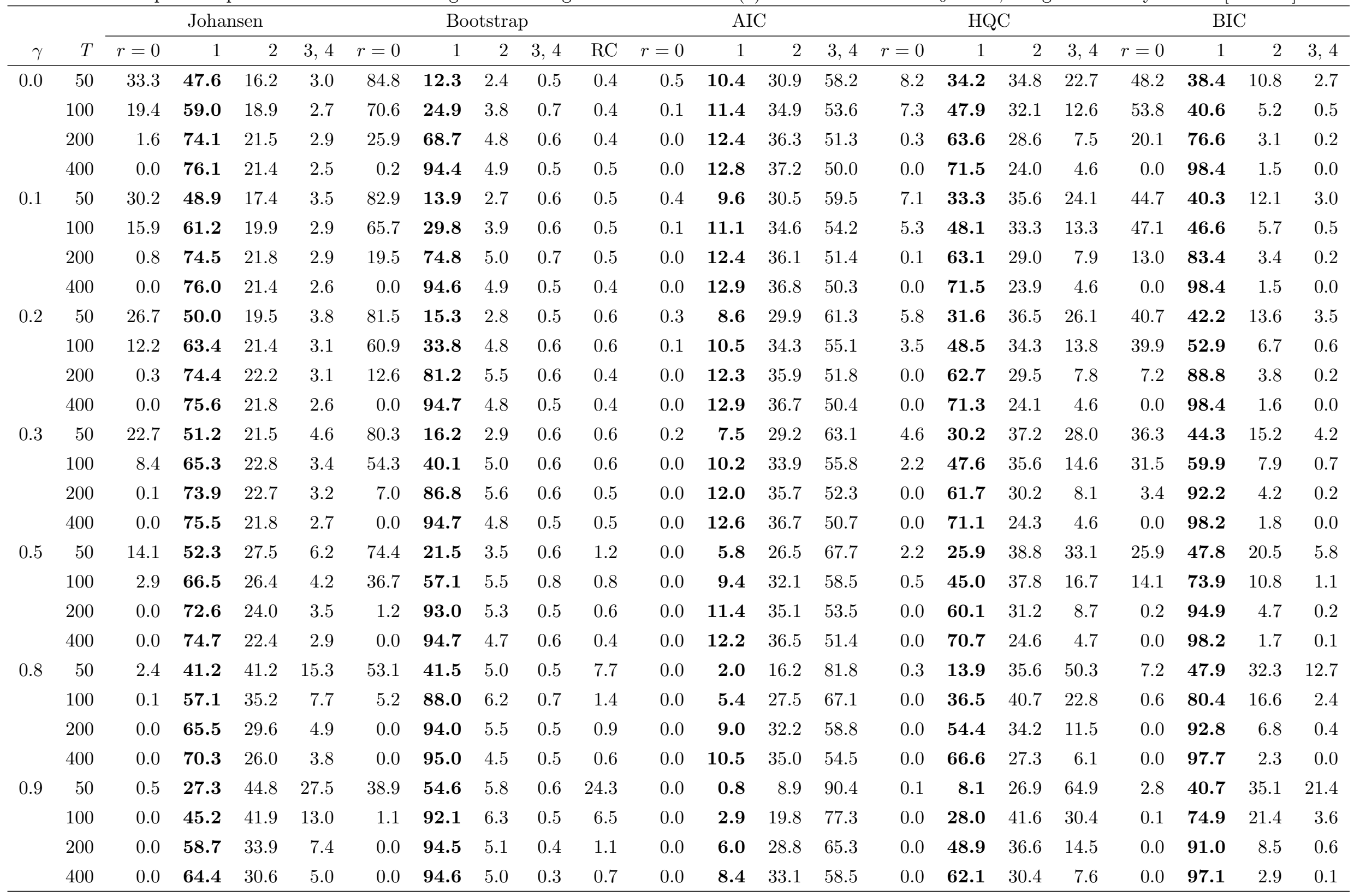


TABLE 3.1: Sequential procedures for determining the co-integration rank. VAR(2) model with rank $r_{0}=2$, i.i.d. Gaussian errors [Case A]

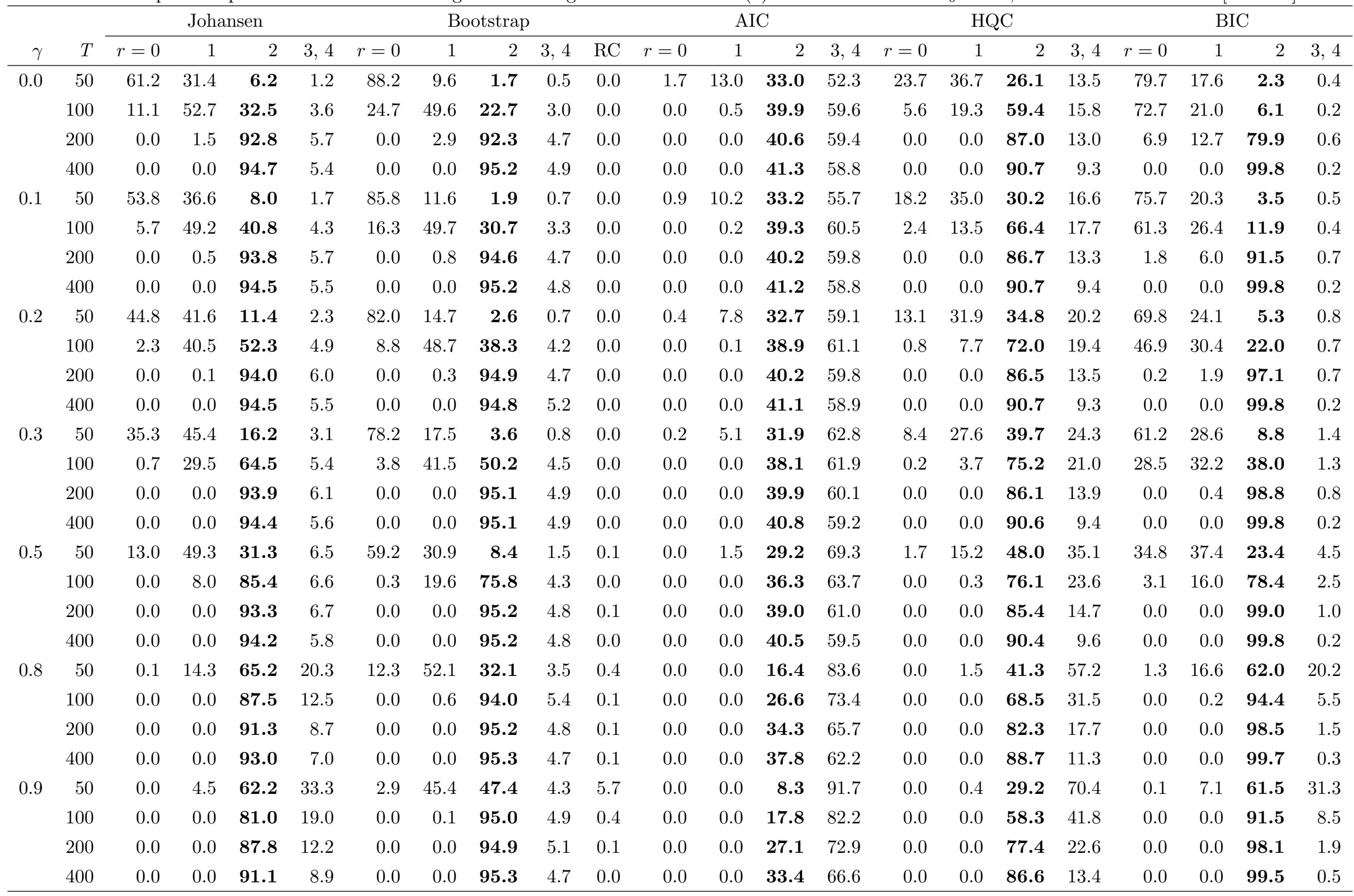


TABLE 3.2: Sequential procedures for determining the co-integration rank. VAR(2) model with rank $r_{0}=2$, i.i.d. $t(5)$ errors [Case B]

\begin{tabular}{|c|c|c|c|c|c|c|c|c|c|c|c|c|c|c|c|c|c|c|c|c|c|c|}
\hline \multirow[b]{2}{*}{$\gamma$} & \multirow[b]{2}{*}{$T$} & \multicolumn{4}{|c|}{ Johansen } & \multicolumn{5}{|c|}{ Bootstrap } & \multicolumn{4}{|c|}{ AIC } & \multicolumn{4}{|c|}{ HQC } & \multicolumn{4}{|c|}{$\mathrm{BIC}$} \\
\hline & & $r=0$ & 1 & 2 & 3,4 & $r=0$ & 1 & 2 & 3,4 & $\mathrm{RC}$ & $r=0$ & 1 & 2 & 3,4 & $r=0$ & 1 & 2 & 3,4 & $r=0$ & 1 & 2 & 3,4 \\
\hline \multirow[t]{3}{*}{0.0} & 50 & 60.0 & 32.4 & 6.6 & 1.1 & 88.8 & 9.2 & 1.7 & 0.3 & 0.0 & 1.5 & 12.2 & 33.3 & 53.0 & 22.2 & 37.1 & 26.7 & 14.0 & 79.0 & 18.1 & 2.6 & 0.4 \\
\hline & 100 & 9.9 & 52.8 & 33.6 & 3.8 & 26.2 & 47.5 & 23.1 & 3.2 & 0.0 & 0.0 & 0.5 & 40.0 & 59.6 & 5.3 & 18.6 & 60.0 & 16.1 & 72.2 & 20.5 & 7.1 & 0.3 \\
\hline & 400 & 0.0 & 0.0 & 94.6 & 5.4 & 0.0 & 0.0 & 95.1 & 4.9 & 0.0 & 0.0 & 0.0 & 41.3 & 58.7 & 0.0 & 0.0 & 90.2 & 9.8 & 0.0 & 0.0 & 99.8 & 0.2 \\
\hline \multirow[t]{3}{*}{0.1} & 50 & 53.0 & 36.2 & 9.1 & 1.6 & 85.5 & 11.7 & 2.3 & 0.5 & 0.0 & 0.9 & 9.6 & 32.4 & 57.1 & 17.9 & 35.2 & 29.9 & 17.0 & 74.7 & 21.0 & 3.6 & 0.7 \\
\hline & 100 & 5.4 & 48.1 & 42.3 & 4.2 & 16.9 & 48.3 & 31.1 & 3.7 & 0.0 & 0.0 & 0.2 & 38.9 & 60.9 & 2.2 & 12.5 & 67.6 & 17.7 & 60.0 & 26.5 & 12.8 & 0.7 \\
\hline & 400 & 0.0 & 0.0 & 94.6 & 5.4 & 0.0 & 0.0 & 95.1 & 4.9 & 0.0 & 0.0 & 0.0 & 42.2 & 57.8 & 0.0 & 0.0 & 90.4 & 9.6 & 0.0 & 0.0 & 99.8 & 0.2 \\
\hline \multirow[t]{4}{*}{0.2} & 50 & 43.7 & 42.6 & 11.2 & 2.6 & 83.5 & 13.2 & 2.7 & 0.6 & 0.0 & 0.5 & 6.9 & 32.6 & 60.1 & 12.4 & 31.6 & 35.2 & 20.8 & 68.4 & 25.1 & 5.6 & 1.0 \\
\hline & 100 & 2.0 & 38.8 & 54.0 & 5.2 & 10.0 & 47.1 & 39.1 & 3.8 & 0.0 & 0.0 & 0.0 & 39.1 & 60.9 & 0.7 & 7.4 & 72.3 & 19.7 & 45.4 & 31.1 & 22.4 & 1.1 \\
\hline & 200 & 0.0 & 0.1 & 93.9 & 6.0 & 0.0 & 0.4 & 95.1 & 4.4 & 0.0 & 0.0 & 0.0 & 40.0 & 60.0 & 0.0 & 0.0 & 86.0 & 14.0 & 0.2 & 2.1 & 97.0 & 0.8 \\
\hline & 400 & 0.0 & 0.0 & 94.1 & 5.9 & 0.0 & 0.0 & 95.3 & 4.7 & 0.1 & 0.0 & 0.0 & 42.4 & 57.6 & 0.0 & 0.0 & 90.2 & 9.8 & 0.0 & 0.0 & 99.7 & 0.3 \\
\hline 0.3 & 50 & 33.0 & 48.1 & 15.6 & 3.3 & 77.8 & 17.6 & 3.9 & 0.7 & 0.0 & 0.1 & 4.9 & 31.5 & 63.5 & 7.4 & 27.2 & 40.3 & 25.1 & 59.8 & 30.0 & 8.6 & 1.6 \\
\hline \multirow{3}{*}{0.5} & 100 & 0.0 & 7.0 & 85.6 & 7.4 & 0.6 & 19.0 & 76.0 & 4.4 & 0.1 & 0.0 & 0.0 & 35.7 & 64.3 & 0.0 & 0.4 & 76.1 & 23.5 & 3.1 & 14.7 & 79.1 & 3.2 \\
\hline & 200 & 0.0 & 0.0 & 93.9 & 6.1 & 0.0 & 0.0 & 95.1 & 4.9 & 0.1 & 0.0 & 0.0 & 40.8 & 59.2 & 0.0 & 0.0 & 85.4 & 14.6 & 0.0 & 0.0 & 99.2 & 0.8 \\
\hline & 400 & 0.0 & 0.0 & 94.2 & 5.9 & 0.0 & 0.0 & 95.2 & 4.8 & 0.0 & 0.0 & 0.0 & 41.4 & 58.6 & 0.0 & 0.0 & 90.3 & 9.7 & 0.0 & 0.0 & 99.6 & 0.4 \\
\hline \multirow[t]{4}{*}{0.8} & 50 & 0.2 & 13.0 & 65.9 & 20.9 & 12.0 & 52.0 & 32.4 & 3.6 & 0.6 & 0.0 & 0.1 & 15.8 & 84.2 & 0.0 & 1.3 & 41.3 & 57.5 & 1.2 & 15.8 & 63.0 & 20.0 \\
\hline & 100 & 0.0 & 0.1 & 86.9 & 13.0 & 0.0 & 0.8 & 94.8 & 4.3 & 0.2 & 0.0 & 0.0 & 27.9 & 72.1 & 0.0 & 0.0 & 68.2 & 31.8 & 0.0 & 0.1 & 94.0 & 5.9 \\
\hline & 200 & 0.0 & 0.0 & 91.0 & 9.0 & 0.0 & 0.0 & 95.3 & 4.7 & 0.1 & 0.0 & 0.0 & 33.7 & 66.3 & 0.0 & 0.0 & 81.8 & 18.2 & 0.0 & 0.0 & 98.5 & 1.5 \\
\hline & 400 & 0.0 & 0.0 & 93.1 & 6.9 & 0.0 & 0.0 & 95.3 & 4.7 & 0.1 & 0.0 & 0.0 & 38.8 & 61.2 & 0.0 & 0.0 & 88.7 & 11.3 & 0.0 & 0.0 & 99.6 & 0.4 \\
\hline \multirow[t]{4}{*}{0.9} & 50 & 0.0 & 4.0 & 62.5 & 33.6 & 3.6 & 43.5 & 49.0 & 4.0 & 5.6 & 0.0 & 0.0 & 8.3 & 91.7 & 0.0 & 0.4 & 29.2 & 70.4 & 0.1 & 6.3 & 61.7 & 32.0 \\
\hline & 100 & 0.0 & 0.0 & 81.0 & 19.0 & 0.0 & 0.2 & 95.4 & 4.4 & 0.5 & 0.0 & 0.0 & 18.4 & 81.6 & 0.0 & 0.0 & 59.0 & 41.0 & 0.0 & 0.0 & 90.7 & 9.3 \\
\hline & 200 & 0.0 & 0.0 & 87.6 & 12.4 & 0.0 & 0.0 & 95.3 & 4.7 & 0.1 & 0.0 & 0.0 & 28.1 & 71.9 & 0.0 & 0.0 & 77.1 & 23.0 & 0.0 & 0.0 & 97.6 & 2.4 \\
\hline & 400 & 0.0 & 0.0 & 91.5 & 8.5 & 0.0 & 0.0 & 95.4 & 4.6 & 0.1 & 0.0 & 0.0 & 34.0 & 66.0 & 0.0 & 0.0 & 86.8 & 13.3 & 0.0 & 0.0 & 99.5 & 0.6 \\
\hline
\end{tabular}


TABLE 3.3: Sequential procedures for determining the co-integration rank. $\operatorname{VAR}(2)$ model with rank $r_{0}=2$, GARCH(1,1) errors [Case C]

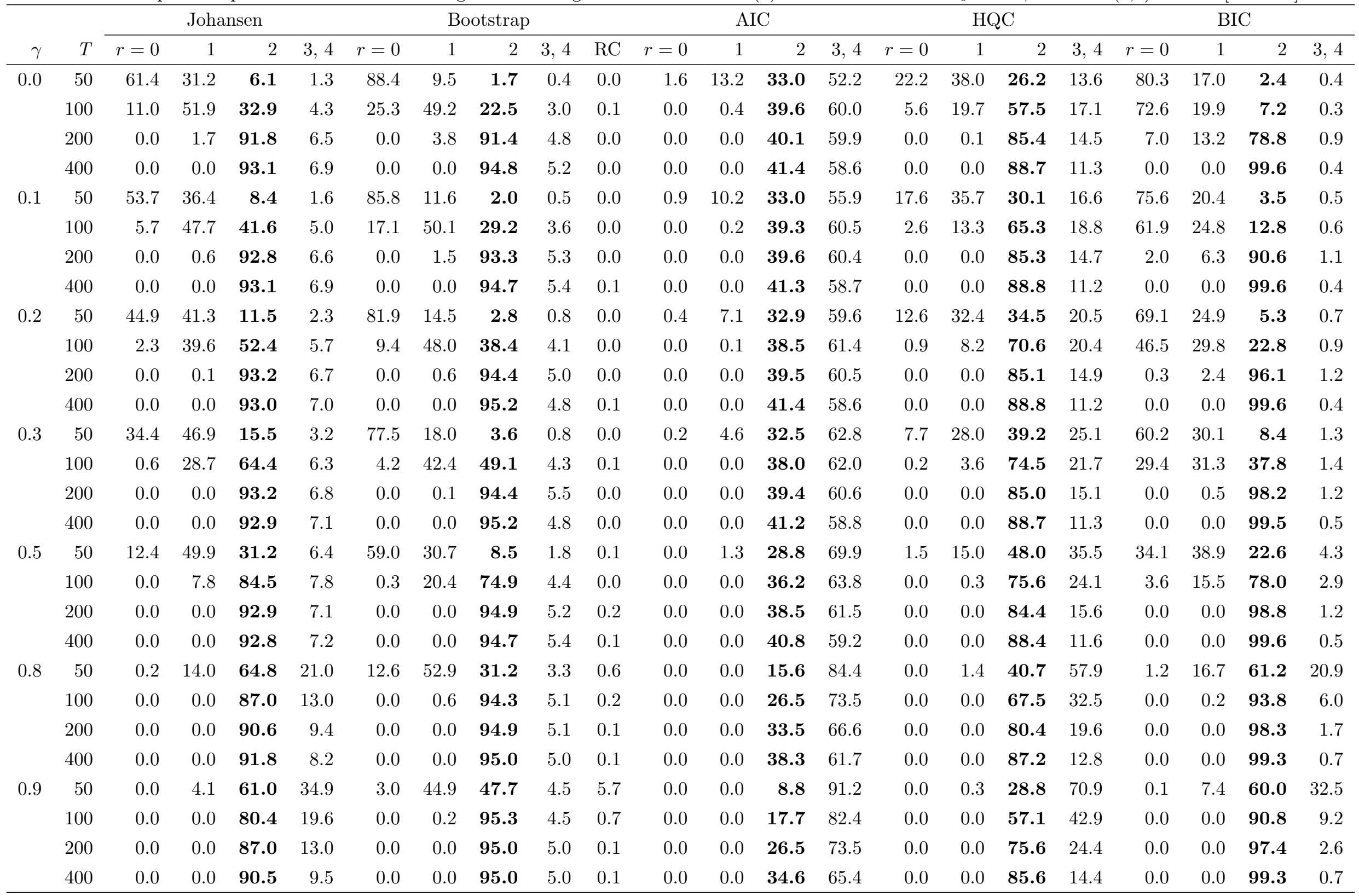


TABLE 3.4: Sequential procedures for determining the co-integration rank. VAR(2) model with rank $r_{0}=2$, Autoregressive Stochastic Volatility [Case D]

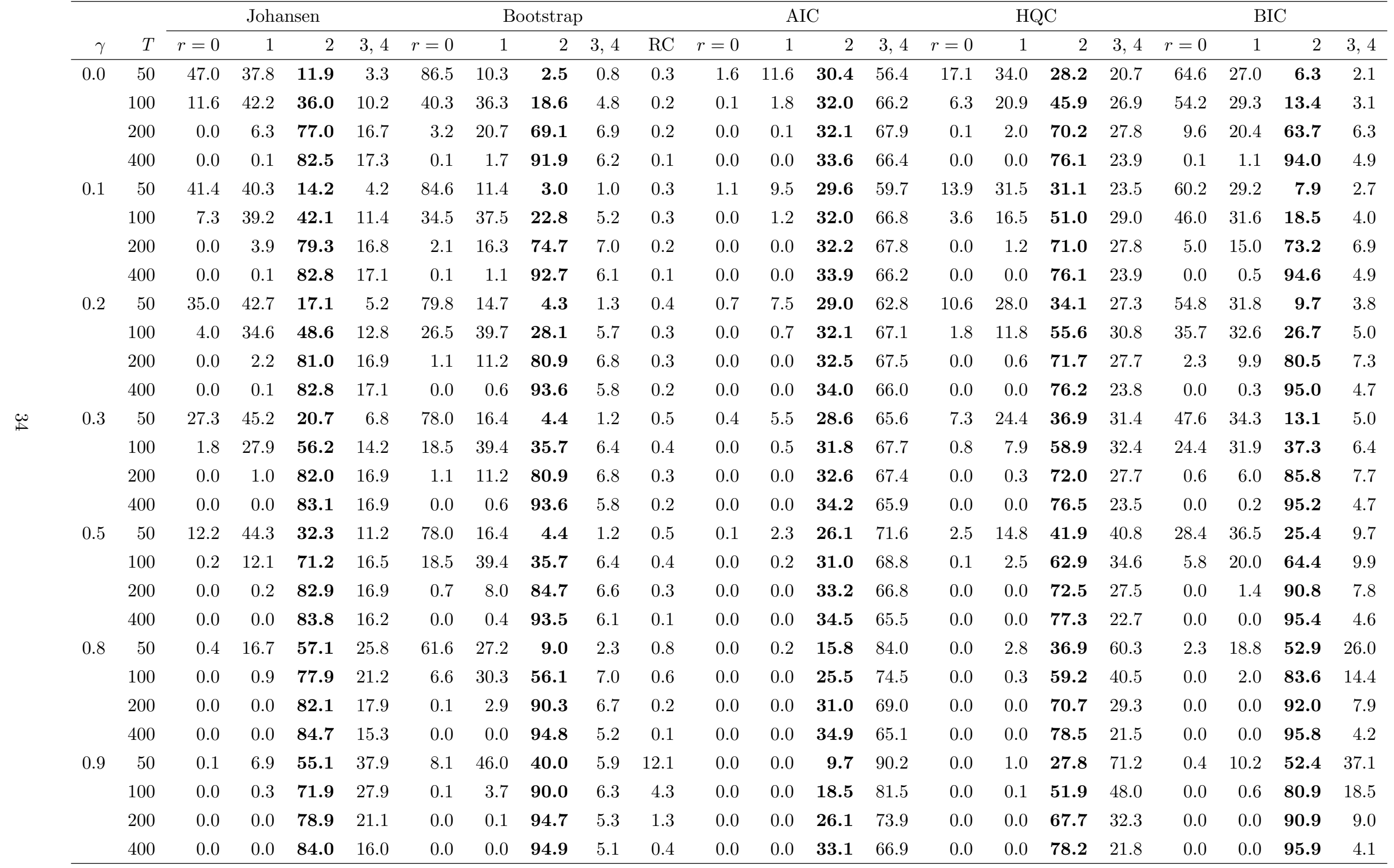


TABLE 3.5: Sequential procedures for determining the co-integration rank. VAR(2) model with rank $r_{0}=2$, Single Volatility Break [Case E]

\begin{tabular}{|c|c|c|c|c|c|c|c|c|c|c|c|c|c|c|c|c|c|c|c|c|c|c|}
\hline \multirow[b]{2}{*}{$\gamma$} & \multirow[b]{2}{*}{$T$} & \multicolumn{4}{|c|}{ Johansen } & \multicolumn{5}{|c|}{ Bootstrap } & \multicolumn{4}{|c|}{ AIC } & \multicolumn{4}{|c|}{ HQC } & \multicolumn{4}{|c|}{ BIC } \\
\hline & & $r=0$ & 1 & 2 & 3,4 & $r=0$ & 1 & 2 & 3,4 & $\mathrm{RC}$ & $r=0$ & 1 & 2 & 3,4 & $r=0$ & 1 & 2 & 3,4 & $r=0$ & 1 & 2 & 3,4 \\
\hline \multirow[t]{3}{*}{0.0} & 50 & 23.7 & 48.9 & 23.0 & 4.3 & 75.6 & 18.6 & 4.7 & 1.1 & 0.4 & 0.2 & 5.8 & 29.1 & 64.9 & 4.9 & 25.9 & 39.2 & 30.1 & 39.5 & 39.8 & 16.5 & 4.2 \\
\hline & 100 & 3.1 & 38.6 & 50.1 & 8.2 & 34.3 & 43.9 & 18.3 & 3.5 & 0.7 & 0.0 & 0.8 & 29.8 & 69.4 & 1.2 & 13.9 & 55.7 & 29.2 & 32.7 & 39.8 & 24.3 & 3.2 \\
\hline & 400 & 0.0 & 0.0 & 87.3 & 12.7 & 0.0 & 0.0 & 95.1 & 4.9 & 0.5 & 0.0 & 0.0 & 30.3 & 69.7 & 0.0 & 0.0 & 78.8 & 21.2 & 0.0 & 0.0 & 98.2 & 1.8 \\
\hline \multirow[t]{3}{*}{0.1} & 50 & 19.9 & 49.3 & 25.9 & 5.0 & 73.6 & 19.8 & 5.3 & 1.3 & 0.4 & 0.1 & 4.7 & 28.4 & 66.8 & 4.0 & 23.0 & 40.7 & 32.3 & 35.4 & 40.5 & 18.9 & 5.2 \\
\hline & 100 & 1.9 & 33.5 & 55.5 & 9.2 & 27.1 & 46.9 & 22.2 & 3.8 & 0.4 & 0.0 & 0.5 & 29.5 & 70.0 & 0.7 & 10.8 & 58.0 & 30.6 & 25.2 & 39.7 & 31.1 & 3.9 \\
\hline & 400 & 0.0 & 0.0 & 87.3 & 12.7 & 0.0 & 0.0 & 95.0 & 5.0 & 0.5 & 0.0 & 0.0 & 30.4 & 69.6 & 0.0 & 0.0 & 78.8 & 21.2 & 0.0 & 0.0 & 98.1 & 1.9 \\
\hline \multirow[t]{4}{*}{0.2} & 50 & 16.6 & 48.2 & 29.3 & 5.9 & 69.7 & 22.3 & 6.4 & 1.6 & 0.6 & 0.1 & 3.9 & 27.7 & 68.3 & 3.2 & 20.4 & 41.6 & 34.8 & 30.8 & 41.0 & 21.9 & 6.3 \\
\hline & 100 & 0.9 & 28.4 & 60.4 & 10.3 & 19.3 & 48.9 & 27.5 & 4.3 & 0.7 & 0.0 & 0.3 & 29.6 & 70.1 & 0.3 & 7.9 & 59.7 & 32.2 & 17.8 & 38.6 & 38.9 & 4.8 \\
\hline & 200 & 0.0 & 0.5 & 86.4 & 13.1 & 0.0 & 7.7 & 87.1 & 5.2 & 0.6 & 0.0 & 0.0 & 30.0 & 70.0 & 0.0 & 0.0 & 73.0 & 27.1 & 0.3 & 5.0 & 90.7 & 4.0 \\
\hline & 400 & 0.0 & 0.0 & 87.1 & 12.9 & 0.0 & 0.0 & 94.8 & 5.2 & 0.5 & 0.0 & 0.0 & 30.4 & 69.6 & 0.0 & 0.0 & 78.8 & 21.2 & 0.0 & 0.0 & 98.0 & 2.0 \\
\hline 0.3 & 50 & 12.6 & 47.3 & 33.4 & 6.8 & 65.0 & 26.2 & 7.0 & 1.8 & 0.6 & 0.0 & 3.2 & 27.0 & 69.9 & 2.2 & 17.3 & 42.4 & 38.1 & 25.5 & 41.2 & 25.5 & 7.8 \\
\hline \multirow{3}{*}{0.5} & 100 & 0.1 & 9.3 & 76.7 & 14.0 & 2.8 & 36.9 & 55.0 & 5.3 & 1.2 & 0.0 & 0.0 & 27.9 & 72.1 & 0.0 & 1.3 & 63.0 & 35.7 & 2.0 & 19.1 & 70.4 & 8.5 \\
\hline & 200 & 0.0 & 0.0 & 85.9 & 14.1 & 0.0 & 0.3 & 94.6 & 5.2 & 1.0 & 0.0 & 0.0 & 30.2 & 69.8 & 0.0 & 0.0 & 72.3 & 27.7 & 0.0 & 0.1 & 95.0 & 4.9 \\
\hline & 400 & 0.0 & 0.0 & 86.6 & 13.4 & 0.0 & 0.0 & 95.0 & 5.0 & 0.8 & 0.0 & 0.0 & 30.5 & 69.5 & 0.0 & 0.0 & 78.7 & 21.3 & 0.0 & 0.0 & 97.8 & 2.2 \\
\hline \multirow[t]{4}{*}{0.8} & 50 & 0.3 & 19.1 & 58.9 & 21.8 & 12.6 & 52.9 & 31.2 & 3.3 & 0.6 & 0.0 & 0.2 & 16.9 & 82.9 & 0.0 & 3.0 & 37.9 & 59.1 & 2.1 & 21.4 & 52.9 & 23.6 \\
\hline & 100 & 0.0 & 0.5 & 79.9 & 19.6 & 0.0 & 0.6 & 94.3 & 5.1 & 0.2 & 0.0 & 0.0 & 23.0 & 77.1 & 0.0 & 0.0 & 57.5 & 42.5 & 0.0 & 1.3 & 85.8 & 13.0 \\
\hline & 200 & 0.0 & 0.0 & 83.4 & 16.6 & 0.0 & 0.0 & 94.9 & 5.1 & 0.1 & 0.0 & 0.0 & 27.0 & 73.1 & 0.0 & 0.0 & 69.2 & 30.8 & 0.0 & 0.0 & 94.1 & 5.9 \\
\hline & 400 & 0.0 & 0.0 & 85.2 & 14.8 & 0.0 & 0.0 & 95.0 & 5.0 & 0.1 & 0.0 & 0.0 & 28.7 & 71.3 & 0.0 & 0.0 & 76.9 & 23.1 & 0.0 & 0.0 & 97.4 & 2.6 \\
\hline \multirow[t]{4}{*}{0.9} & 50 & 0.0 & 9.6 & 58.6 & 31.8 & 3.0 & 44.9 & 47.7 & 4.5 & 5.7 & 0.0 & 0.0 & 11.9 & 88.0 & 0.0 & 1.1 & 30.8 & 68.1 & 0.3 & 12.3 & 54.6 & 32.8 \\
\hline & 100 & 0.0 & 0.1 & 75.6 & 24.4 & 0.0 & 0.2 & 95.3 & 4.5 & 0.7 & 0.0 & 0.0 & 18.7 & 81.3 & 0.0 & 0.0 & 51.8 & 48.2 & 0.0 & 0.2 & 84.1 & 15.8 \\
\hline & 200 & 0.0 & 0.0 & 80.7 & 19.3 & 0.0 & 0.0 & 95.0 & 5.0 & 0.1 & 0.0 & 0.0 & 23.5 & 76.5 & 0.0 & 0.0 & 65.8 & 34.2 & 0.0 & 0.0 & 92.9 & 7.1 \\
\hline & 400 & 0.0 & 0.0 & 83.6 & 16.4 & 0.0 & 0.0 & 95.0 & 5.0 & 0.1 & 0.0 & 0.0 & 25.9 & 74.1 & 0.0 & 0.0 & 74.4 & 25.6 & 0.0 & 0.0 & 96.9 & 3.1 \\
\hline
\end{tabular}

